\title{
Driven Quantum Dynamics: Will It Blend?
}

\author{
Leonardo Banchi, ${ }^{1}$ Daniel Burgarth, ${ }^{2}$ and Michael J. Kastoryano ${ }^{3}$ \\ ${ }^{1}$ Department of Physics and Astronomy, University College London, \\ Gower Street, WCIE 6BT London, United Kingdom \\ ${ }^{2}$ Institute of Mathematics, Physics, and Computer Science, Aberystwyth University, \\ Aberystwyth SY23 2BZ, United Kingdom \\ ${ }^{3}$ NBIA, Niels Bohr Institute, University of Copenhagen, DK-2100 Copenhagen, Denmark
} (Received 4 May 2017; revised manuscript received 19 September 2017; published 20 October 2017)

\begin{abstract}
Randomness is an essential tool in many disciplines of modern sciences, such as cryptography, black hole physics, random matrix theory, and Monte Carlo sampling. In quantum systems, random operations can be obtained via random circuits thanks to so-called $q$-designs and play a central role in condensedmatter physics and in the fast scrambling conjecture for black holes. Here, we consider a more physically motivated way of generating random evolutions by exploiting the many-body dynamics of a quantum system driven with stochastic external pulses. We combine techniques from quantum control, open quantum systems, and exactly solvable models (via the Bethe ansatz) to generate Haar-uniform random operations in driven many-body systems. We show that any fully controllable system converges to a unitary $q$-design in the long-time limit. Moreover, we study the convergence time of a driven spin chain by mapping its random evolution into a semigroup with an integrable Liouvillian and finding its gap. Remarkably, we find via Bethe-ansatz techniques that the gap is independent of $q$. We use mean-field techniques to argue that this property may be typical for other controllable systems, although we explicitly construct counterexamples via symmetry-breaking arguments to show that this is not always the case. Our findings open up new physical methods to transform classical randomness into quantum randomness, via a combination of quantum many-body dynamics and random driving.
\end{abstract}

DOI: 10.1103/PhysRevX.7.041015

\section{INTRODUCTION}

Randomness generating quantum operations play a central role in our understanding of many various physical phenomena [1]. Recently, with the development of quantum information processing, random operations have found new applications, not only as a theoretical tool but also in practical protocols. Indeed, they are used in quantum cryptography [2], quantum process tomography [3], fidelity estimation [4], quantum communication and entanglement sharing [5-7], quantum data hiding [2,8,9], and entanglement generation [10-13]. Because of their crucial importance, several procedures have been developed to generate either truly random or pseudorandom operations via random quantum circuits [4,14-20]. However, from the physical point of view, these protocols often have a complexity comparable with universal quantum computation, being based on the application of a sufficiently large set of quantum gates. Here, on the other hand, we consider

Published by the American Physical Society under the terms of the Creative Commons Attribution 4.0 International license. Further distribution of this work must maintain attribution to the author(s) and the published article's title, journal citation, and DOI.
Subject Areas: Quantum Physics,

Quantum Information,

Statistical Physics a more physically inspired approach, based on quantum control, where the quantum system is controlled by random classical pulses.

Quantum control is an established research field at the overlap of control theory and quantum mechanics. Essentially it provides a framework to steer a quantum system through Hilbert space by applying time-dependent fields. Controllability is a powerful algebraic tool to fully characterize when any possible unitary evolution in the system's Hilbert space can be obtained from the Schrödinger equation with a suitable choice of timedependent fields. The central question of this paper is what happens when we apply random fields to a controllable system. We show, under some conditions, that after a suitably long mixing time the corresponding random unitary evolutions of the system converge to a uniformly random set, as measured by the Haar measure. Therefore, one of the central result of this paper is that driving a controllable quantum system with stochastic control pulses offers a natural approach to generate random unitary operations with physical processes.

Within this picture, the estimation of the mixing time is the crucial theoretical aspect. We use several tools from the theory of open quantum systems and many-body physics, such as low-energy effective Liouvillians, mean-field 
techniques, and the Bethe ansatz, to find an accurate estimation of the mixing time in several situations. In particular, we focus on a one-dimensional system with edge control due to the availability of analytical tools, as well as the intuitive interpretation available in such a system with Lieb-Robinson bounds and spin waves. This particular case is also motivated by the current experimental capabilities in integrated photonic circuits [21,22], where different stochastic control pulses can be simulated by changing the spatial extent of the waveguides via electrically tuned onchip heaters [23]. In those systems a major recent result has been the experimental measurement of boson sampling [24-26], a problem that is believed to be hard to simulate classically. Random unitary operations and higher-dimensional systems are required in boson sampling to have a convincing demonstration of quantum computational supremacy [27]. Pseudorandom operations in those experiments are currently obtained via a finely tuned network of several beam splitters and phase shifters. The different approach we present here is based on the simpler implementation of noisy quantum walks and, therefore, can offer an advantage to perform boson sampling experiments on larger scale.

A further motivation for this paper comes from quantum control itself. The algebraic tools developed in quantum control are typically not able to provide an estimation of the control time needed to reach a given target operation. In view of practical applications, this is a big handicap, because noise will always limit the total time available to an experimenter. It is therefore of interest to find estimates of such times. The analytical expressions for the mixing time obtained in this paper also provide an easily computable upper bound for the control time. Indeed, by definition, after the mixing time the system has already explored all possible unitary evolutions with stochastic control pulses. This implies that, apart from measure zero sets, at this time any evolution is achievable with a suitable choice of the control field.

Finally, another motivation for the present work is for the problem of fast scrambling of quantum information. The problem was first identified in the setting of black hole physics $[28,29]$, where it was conjectured that black holes start evaporating information when most localized microscopic degrees of freedom (d.o.f.) become inaccessible without measuring a constant fraction of the whole system. Unfortunately, identifying mechanisms for fast scrambling has been challenging, and providing tools to rigorously analyze scrambling times even more so. Moreover, explicit constructions of fast scramblers [30] are not directly inspired by physical models. Here, we describe a physically motivated process that could lead to new insights in the design and analysis of fast scrambling models.

The paper is organized as follows. In Sec. II, we show how to obtain Haar-uniform unitary evolutions (i.e., a unitary design) via quantum control techniques. We focus on $q$-design, not only for its applications in quantum information, but also to quantify the distance with the target uniform distribution. We consider Markovian stochastic control pulses and introduce some general techniques for the estimation of the mixing time. In Sec. III, we map the problem of unitary design to a general many-body problem, studying its mean-field solution and discussing the limitations of the latter approach via symmetry-breaking arguments. In Sec. IV, we focus on a specific onedimensional model controlled at one of its boundaries. We show that this model in certain limits can be mapped to an exactly solvable model and we study its analytic solution via Bethe-ansatz techniques. A central result of this section is that the mixing time for this particular model is independent of the number of copies $q$. Intuitively, the $q$ independence implies that pseudorandom unitaries obtained with random control pulses approximate all the moments of the Haar distribution with the same accuracy. These predictions are then corroborated with numerical simulations. In Sec. V, we show other applications for boson sampling, the decay of correlations in spin chains, and the estimation of the control time. Conclusions and perspectives are written in Sec. VI.

\section{UNITARY DESIGNS VIA QUANTUM CONTROL}

Physical quantum systems are modeled via a Hamiltonian operator $H$, which describes the interactions between the components of the system. When external control is applied to the system, its evolution is represented by a time-dependent Hamiltonian,

$$
\hat{H}(t)=H+g(t) V,
$$

where $g(t)$ is an external control pulse and $V$ is an operator. If $d$ is the dimension of the Hilbert space, then $H$ and $V$ are $d \times d$ Hermitian matrices while $g(t)$ is a scalar function depending on time $t$. For multiple pulses, $\hat{H}(t)=$ $H+\sum_{i} g_{i}(t) V_{i}$. After some time $T$, the combined action of the natural interactions and the external pulses is a unitary operation $U=\mathcal{T} \exp \left[-i \int_{0}^{T} \hat{H}(s) d s\right]$, where $\mathcal{T}$ represents the time order operator. In general, the amount of different unitary operations $U$ that can be obtained from the dynamics of the system is limited. However, if the system is fully controllable, then any operation can be obtained with a suitable engineering of the control pulse. In other terms, given any $U \in \mathrm{SU}(d)$, it is possible to find a control profile $g(t)$ such that $U=\mathcal{T} \exp \left[-i \int_{0}^{T} \hat{H}(s) d s\right]$, where the control time $T$ depends on the target unitary $U$. There are many powerful theorems to test controllability. In general, a system described by the Hamiltonian as in Eq. (1) is controllable [31] if $H, V$ and their nested commutators $[A,[B,[C, \ldots]]]$ (where $\{A, B, C, \ldots\} \in\{H, V\}$ ) generate the Lie algebra of $\mathrm{SU}(d)$. Although the algebraic conditions for controllability are well known, it is still an open 
problem in quantum control to estimate the control time $T$, given also the knowledge of the target gate $U$ and the operators $H$ and $V$. For fully controllable systems there exists a minimal control time, generally unknown, such that all target gates can be obtained exactly at that time [32]. For small dimensional systems, analytic bounds of such universal control time may be found in terms of quantum speed limits or Cartan decompositions of spin systems. In a high-dimensional system, such tools become intractable. If the system is drift free $\left(H_{0}=0\right)$, control times are trivial or determined only by energy bounds on the time-dependent fields. We are instead interested in systems where the controls need to work together with a drift to achieve full control (so-called weak controllability). In such a case, the time scale is bounded by the dynamics of the drift and provides insights into the many-body physics triggered by it.

We now consider the control pulse as a stochastic process, namely where a certain profile $g(t)$ can be applied to the system with a probability $p_{g(t)}$, and study the distribution of the resulting unitary operations. Such a random pulse can be obtained, for example, by considering the Fourier expansion of the control signal,

$$
g(t)=\sum_{k=1}^{K} A_{k} \cos \left(\omega_{k} t+\varphi_{k}\right),
$$

where the amplitudes $A_{k}$, the phases $\varphi_{k}$, and possibly even the frequencies $\omega_{k}$ are random variables. We use the notation $\mathbb{E}[\cdot]$ to denote the average over those random variables. Repeating the experiment with many random signals, one obtains a distribution of unitary matrices, where each matrix $U$ is obtained with probability $p_{U}$. Random unitary operations play a central part in many quantum information protocols. A pivotal role in many applications is played by the uniform distribution, also called the Haar distribution, which is invariant under the action of the unitary group itself. In the following sections we study when, and how rapidly, the distribution $p_{U}$ converges to the Haar-uniform distribution.

\section{A. Comparing random evolutions: Unitary $q$-design}

Obtaining truly uniform random unitaries is a very hard task, and normally one observes pseudouniform distributions which approximate the uniform (Haar) measure up to some errors. Pseudouniform distributions can be obtained with random quantum circuits [4,14-18], but these circuits typically require many different gates that make the implementation in physical systems demanding. Recently, alternative protocols based on physically inspired time-dependent Hamiltonians have been proposed [33,34]. Nonetheless, these approaches still require that all the interactions inside the system should change in time, an assumption that currently is beyond reach in many experimental platforms. Here, on the other hand, we focus on a general scheme which occurs in most quantum systems, namely, when the natural and time-independent interaction $H$ experienced by the system is paired with an external control, as in Eq. (1).

There are many ways of comparing the distance between two quantum processes. When dealing with randomness generating processes, it is often convenient and relevant to work with approximate $q$-designs [35]. A unitary $q$-design is a distribution of unitaries, possibly discrete, that gives the same expectations of the Haar distribution for polynomial functions of degree at most $q$ (see, e.g., Ref. [36]). It is often inaccessible experimentally to distinguish between truly random processes and approximate $q$-designs. Formally, approximate $q$-designs are defined by the requirement that

$\left\|\mathbb{E}_{U}\left[U^{\otimes q}(\cdot) U^{\otimes q^{\dagger}}\right]-\int U^{\otimes q}(\cdot) U^{\otimes q \dagger} \mu_{\text {Haar }}(d U)\right\|_{\diamond}<\epsilon$,

for suitably small $\epsilon$, where $\|\cdot\|_{\diamond}$ refers to the diamond norm, $\mathbb{E}_{U}$ denotes an average over some given distribution of unitaries $\mu_{U}$, and $\mu_{\text {Haar }}(d U)$ is the Haar measure. This is the most stringent distinguishability measure between quantum processes, and guarantees that no single (global) measurement on the system and a possible ancilla can distinguish between the two processes with probability larger than $\epsilon$. A related notion [18] is that of quantum expanders, which are defined by

$e\left(\mu_{U}, q\right)=\left\|\mathbb{E}_{U}\left[U^{\otimes q, q}\right]-\int U^{\otimes q, q} \mu_{\text {Haаr }}(d U)\right\|_{\infty}<\epsilon$,

where $X^{\otimes q, q}=X^{\otimes q} \otimes\left(X^{\otimes q}\right)^{*}$. Equation (4) can be regarded as the vectorized version of Eq. (3): given an operator $X=\sum_{i j} X_{i j}|i\rangle\langle j|$, its vectorized form is $\left.|X\rangle\right\rangle=\sum_{i j} X_{i j}|i j\rangle$. However, it is strictly weaker, and the separation between the two bounds can be exponential in the system size. However, Eq. (4) is often much easier to work with in practice [18]. It follows from the definition that $|A X\rangle\rangle=$ $A \otimes \mathbb{1}|X\rangle\rangle$ and $\left.|X A\rangle\rangle=\mathbb{1} \otimes A^{T}|X\rangle\right\rangle$. Therefore, $X^{\otimes q, q}$ is the vectorization of the superoperator $\rho \mapsto X^{\otimes q} \rho X^{\otimes q^{\dagger}}$. Quantum expanders and $q$-design compare probability distributions of unitary matrices by comparing the "moments" of the distribution, namely, random processes that depend polynomially on the random variable. Two close distributions of unitary matrices have similar moments, as shown in Ref. [14], $e(\mu, q) \leq 2 q \mathcal{W}\left(\mu, \mu_{\text {Haar }}\right)$, for all measures $\mu$, with $\mathcal{W}$ the Wasserstein distance [37], $\mathcal{W}\left(\mu_{1}, \mu_{2}\right)=\sup _{f}\left|\int f(U)\left[\mu_{1}(d U)-\mu_{2}(d U)\right]\right|$, where $f$ is a 1-Lipschitz function and $U$ is a unitary matrix. The Wasserstein distance is a measure between classical probability distributions, and hence one can use a number of 
classical Markov chain mixing tricks to bound it. However, we do not use it, as we instead use tools from condensedmatter physics to bound the mixing time.

In the quantum control setting, $\mathbb{E}_{U}$ in Eqs. (3) and (4) is the average over many unitary operations obtained after the application of random pulses up to a certain time $T$. Therefore,

$$
\begin{aligned}
\mathbb{E}_{U} & {\left[U^{\otimes q} \rho U^{\otimes q^{\dagger}}\right] } \\
& =\mathbb{E}\left[\left(\mathcal{T} e^{-i \int_{0}^{T} \hat{H}(s) d s}\right)^{\otimes q} \rho\left(\mathcal{T} e^{i \int_{0}^{T} \hat{H}(s) d s}\right)^{\otimes q}\right] .
\end{aligned}
$$

To simplify the theoretical description of this problem we make two assumptions. (i) We assume that the stochastic process $g(t)$ is Gaussian. This is a reasonable approximation in many cases and can be obtained, e.g., via Eq. (2) when $K \gg 1$, in view of the central limit theorem. (ii) We assume also that $g(t)$ is harmonic, namely, that $\mathbb{E}[g(t+$ $s) g(t)]=c(s)$ is independent of $t$. Moreover, without loss of generality, the harmonic process can be chosen such that $\mathbb{E}[g(t)]=0$. In view of these assumptions, exploiting the results of Refs. [38,39], in Appendix A we find a closedform expression for Eq. (5). That expression can be drastically simplified if we assume that the correlation time is finite and there exists a suitably large $T$ such that $T c(T s) \simeq(\sigma / 2) \delta(s)$, where $\delta$ is the Dirac delta function and $\sigma$ is a constant. In the long-time limit, $t>T \gg$ $\|H\|,\|V\|$, one finds then that

$$
\mathbb{E}_{U}\left[U^{\otimes q} \rho U^{\otimes q^{\dagger}}\right] \simeq e^{-t \mathcal{L}^{q} \rho}
$$

where

$$
\mathcal{L}^{q} \rho=-i\left[H^{\oplus q}, \rho\right]-\frac{\sigma}{2}\left[V^{\oplus q},\left[V^{\oplus q}, \rho\right]\right],
$$

and $X^{\oplus q}=X \oplus X \oplus \ldots$, with $\oplus$ the Kronecker sum $X \oplus Y=X \otimes \mathbb{1}+\mathbb{1} \otimes Y$. Therefore, with these three approximations, the long-time dynamics of the stochastic process is Markovian and described by the above Lindblad equation [40,41], where the operator $\mathcal{L}^{q}$ is called Liouvillian. Similarly to what happens with the replica trick in statistical physics [42], the average over the noise effectively couples the initially uncoupled copies. Sometimes we use the more convenient vectorized form of the above equation,

$$
\mathcal{L}_{q}=-i \stackrel{\circ}{H}^{\oplus q}-\frac{\sigma}{2}\left(\stackrel{\circ}{V}^{\oplus q}\right)^{2},
$$

where $\stackrel{\circ}{X}=X \otimes \mathbb{1}-\mathbb{1} \otimes X^{T}$ is the vectorization of the commutator $[X, \cdot]$. If $t \rightarrow \infty$, then $\mathbb{E}_{U}\left[U^{\otimes q} \rho U^{\otimes q^{\dagger}}\right]$ converges to one of the steady states of the Liouvillian $\mathcal{L}^{q}$.

In the following section, we prove that the steady-state manifold of $\mathcal{L}^{q}$ coincides with the state space after averaging over the Haar measure, namely, that all the moments of the random unitary evolution converge to the averages over the uniform distribution for $t \rightarrow \infty$. Moreover, we study the mixing time via the gap of the Liouvillian and show that, in several cases, the latter is independent of $q$. Physically this is important, because it implies that all the moments converge (in 2-norm) at the same time, as given by the inverse of the Liouvillian gap, and that, accordingly, we can use the latter to estimate the mixing time of the random unitary evolutions.

\section{B. Steady state of the Liouvillian evolution}

We start by describing the steady state of $\mathcal{L}^{q}$. In general, the dimensionality of the steady state set is in one-to-one relation with the conserved quantities of the Lindbladian evolution [43]. Given an orthonormal basis $\left\{M_{\mu}\right\}$ of the steady-state space, equipped with the standard HilbertSchmidt product, there exists a dual operator set $\left\{J_{\mu}\right\}$ such that $\mathcal{L}^{q \dagger} J_{\mu}=0$, where $\mathcal{L}^{q \dagger}$ is the Liouvillian operator Eq. (7) after the substitution $H \rightarrow-H$. The latter substitution does not change the dynamical algebra, so algebraic considerations based on controllability hold also for $\mathcal{L}^{q \dagger}$. From the conserved quantities $J_{\mu}$ and their dual operators $M_{\mu}$ one finds the steady state as $\rho_{\infty}=$ $\sum_{\mu} M_{\mu} \operatorname{Tr}\left(J_{\mu} \rho_{0}\right)$, where $\rho_{0}$ is the initial state [43]. Since the system is controllable, repeated commutators of $H^{\oplus q}$ and $V^{\oplus q}$ give rise to the algebra $\operatorname{su}(d)^{\oplus q}$. Therefore, because of the Schur-Weyl duality [44], the only operators that commute with both $H^{\oplus q}$ and $V^{\oplus q}$, and more generally with Eq. (5), are index permutation operators. Let $S_{q}$ be the group of permutations of the set $1, \ldots, q$ and let $P_{\sigma}, \sigma \in S_{q}$ be the operator that permutes the index of the tensor copy $\mathcal{H}^{\otimes q}$, namely, the operator that maps $\psi_{i_{1}, i_{2}, \ldots, i_{n}}$ to $\psi_{\sigma\left(i_{1}\right), \sigma\left(i_{2}\right), \ldots, \sigma\left(i_{n}\right)}$ for each set of indices $i_{j}$. It is simple to show that $P_{\pi} P_{\sigma}=P_{\pi \sigma}$ and that these operators form a unitary representation of the permutation group $S_{q}$. The index permutation operators are the only conserved quantities of the Liouvillian, $\mathcal{L}^{q}\left(P_{\rho}\right)=\mathcal{L}^{q^{\dagger}}\left(P_{\rho}\right)=0$, so $\rho_{\infty}=\sum_{\sigma} \rho_{\sigma} P_{\sigma}$. However, since the operators $P_{\sigma}$ are not orthonormal, one has

$$
\operatorname{Tr}\left[P_{\sigma}^{\dagger} \rho_{0}\right]=\operatorname{Tr}\left[P_{\sigma}^{\dagger} \rho_{\infty}\right]=\sum_{\pi \in S_{q}} \rho_{\pi} \operatorname{Tr}\left[P_{\sigma}^{\dagger} P_{\pi}\right],
$$

wherein the first equality holds because $P_{\sigma}$ is a conserved quantity. By inverting the above equation, we find that

$$
\rho_{\infty}=\lim _{t \rightarrow \infty} e^{t \mathcal{L}_{q}} \rho_{0}=\sum_{\pi, \sigma}\left(M^{-1}\right)_{\pi \sigma} \operatorname{Tr}\left[P_{\sigma}^{\dagger} \rho_{0}\right] P_{\pi},
$$

where $M_{\sigma \pi}=\operatorname{Tr}\left[P_{\sigma}^{\dagger} P_{\pi}\right]$. It has been shown in Ref. [45] that $M_{\sigma \pi}=d^{l\left(\sigma^{-1} \pi\right)}$, where $l(\sigma)$ is the number of cycles in the cycle decomposition of $\sigma$. The dimensionality of the steady-state manifold is then given by the matrix rank of 
$M$. One finds that the steady-state degeneracy is $\sim e^{\mathcal{O}(q)}$. The right-hand side of Eq. (10) is exactly equal to the integration over the Haar measure (see, e.g., Proposition 3 in Ref. [45]). Therefore, we show that

$$
\lim _{t \rightarrow \infty} e^{t \mathcal{L}^{q}} \rho=\int d U U^{\otimes q} \rho U^{\otimes q^{\dagger}},
$$

namely, that the infinite time evolution of the system under the Liouvillian Eq. (7) is equivalent to an integration over the Haar measure.

In summary, we show that by driving a controllable system with random control pulses Eq. (2), where the stochastic process is Gaussian, harmonic, and has a finite correlation time, the resulting average evolution of the quantum system converges for $t \rightarrow \infty$ to a uniform integration over the Haar measure.

\section{Construction of excited states}

Certain excited states of the Liouvillian Eq. (8) can be built up directly from the excitations of the individual quantum systems. It is convenient to separate $\mathcal{L}_{q}$ from Eq. (8) into local terms $\mathcal{L}_{k}^{\text {loc }}$ acting only on the $k$ th copy, and a nonlocal interaction. Indeed,

$$
\begin{gathered}
\mathcal{L}_{q}=\sum_{k=1}^{q} \mathcal{L}_{k}^{\text {loc }}-\frac{\sigma}{2} \sum_{k \neq l=1}^{q} \stackrel{\circ}{V}_{k} \stackrel{\circ}{V}_{\ell}, \\
\mathcal{L}_{k}^{\text {loc }}=-i \stackrel{\circ}{H}_{k}-\frac{\sigma}{2} \stackrel{\circ}{V}_{k}^{2},
\end{gathered}
$$

where $\stackrel{\circ}{H}_{k}, \stackrel{\circ}{V}_{k}$, and accordingly $\mathcal{L}_{k}^{\text {loc }}$, act only on the $k$ th copy. Therefore, each $\mathcal{L}_{k}^{\text {loc }}$ for different $k$ is equivalent to a single-copy Liouvillian $\mathcal{L}_{1}$. We assume that the operator $\mathcal{L}_{1}$ is diagonalizable (with right and left eigenvectors) and call

$$
\mathcal{L}_{1}=\sum_{i} \lambda_{i} \Pi_{(i)}
$$

its eigenvalue decomposition, where the eigenvalues $\lambda_{j}$ are ordered with decreasing real part (starting from zero) and $\Pi_{j}$ are the corresponding eigenprojections. The operators

$$
\Pi_{j}^{(i)}=\Pi_{(0)}^{\otimes(j-1)} \otimes \Pi_{(i)} \otimes \Pi_{(0)}^{(q-j)}
$$

are then eigenprojections of $\mathcal{L}^{q}$, with eigenvalue $\lambda_{i}$. To show this, we note indeed that $\Pi_{j}^{(i)}$ is proportional to the vectorization of the identity operator in each copy, aside from the $j$ th one, since $\Pi_{(0)}$ is the projection onto the steady state and, accordingly, $\Pi_{(0)}(X)=\rho_{\infty} \operatorname{Tr}[X]$, which is proportional to the identity operator. Therefore, $\stackrel{\circ}{V}_{l} \Pi_{j}^{(i)}=0$ [because $\left[V_{l}, \Pi_{j}^{(i)}(X)\right]=0$ for all $\left.X\right]$, as long as $l \neq j$. On the other hand, for $l=j$, it is $\stackrel{\circ}{V}_{k} \stackrel{\circ}{V}_{l} \Pi_{j}^{(i)}=0$, since by construction $k \neq j$. This shows that Eq. (15) is a projector on the eigenspace of $\mathcal{L}_{q}$ with eigenvalue $\lambda_{i}$. Moreover, from the operators Eq. (15) one can also construct the eigenstates of $\mathcal{L}_{q}$ that act on the irreducible representations of the symmetric group - indeed, since the permutation operators $P_{\sigma}$ commute with the Liouvillian, then $P_{\sigma}\left(\Pi_{j}^{(i)}\right) P_{\sigma}^{\dagger}$ is an eigenprojection of $\mathcal{L}_{q}$ for all $\sigma$.

In summary, the eigenstate of $\mathcal{L}_{1}$ with the lowest gap can be used to construct some exact eigenstates of $\mathcal{L}_{q}$, although it remains to be shown that they have the smallest gap. These eigenvalues have degeneracy at least as large as the ground-state degeneracy, since $P_{\rho} \Pi_{j}^{(i)}$ is also an eigenvector with eigenvalue $\lambda_{j}$ of $\mathcal{L}^{q}$.

\section{Convergence time}

Given the results of the previous section, we want to know how rapidly the semigroup converges to the uniform distribution Eq. (11). In Appendix B, we provide a brief introduction to the convergence theory of dynamical semigroups, and argue that when the generator is not reversible (detailed balance), the convergence is governed by the singular value gap of the channels rather than the spectral gap of the generator. In general, we want to bound the trace norm, but it is more convenient to analyze the $2 \rightarrow 2$ norm:

$$
\left\|e^{t \mathcal{L}^{q}}-\mathcal{U}_{\infty}\right\|_{1 \rightarrow 1} \leq d^{2 q}\left\|e^{t \mathcal{L}^{q}}-\mathcal{U}_{\infty}\right\|_{2 \rightarrow 2}
$$

where $\mathcal{U}_{\infty}=\lim _{t \rightarrow \infty} e^{t \mathcal{L} q}$ and $d$ is the dimension of the local Hilbert space. Let $s_{j}(t)$ be the singular values of $e^{t \mathcal{L}}$, ordered from largest to smallest. The largest has magnitude one. Then the singular values of $\left(e^{t \mathcal{L}}-\mathcal{U}_{\infty}\right)$ are strictly smaller than one, and

$$
\left\|e^{t \mathcal{L}}-\mathcal{U}_{\infty}\right\|_{2 \rightarrow 2}=\sup _{\psi}\left|\left\langle\psi\left|\left(e^{t \hat{\mathcal{L}}} e^{t \hat{\mathcal{L}}^{\dagger}}-\hat{\mathcal{U}}_{\infty}\right)\right| \psi\right\rangle\right| .
$$

If the Liouvillian were reversible, then the singular values $s_{j}(t)$ would be given by $e^{t \lambda_{j}}$, where $\lambda_{j}$ are the eigenvalues of $\mathcal{L}$. Unfortunately, the semigroups that we are working with are not Hermitian. Nonetheless, from Eq. (B5), we find that the $2 \rightarrow 2$ norm can be bounded in terms of the eigenvalues and eigenvectors of $\mathcal{L}^{q}$ as

$$
\left\|e^{t \mathcal{L}^{q}}-\mathcal{U}_{\infty}\right\|_{2 \rightarrow 2} \leq \sum_{j: \lambda_{j} \neq 0} e^{t \operatorname{Re}\left[\lambda_{j}\right]} \sqrt{\left\|R_{j}\right\|\left\|L_{j}\right\|}
$$

where $\lambda_{j}$ are the eigenvalues of $\mathcal{L}^{q}$, and $R_{j}, L_{j}$ are its right and left eigenvectors, satisfying $\operatorname{tr}\left[L_{j}^{\dagger} R_{k}\right]=\delta_{j k}$.

In general it is very difficult to bound Eq. (18), since the norms of the eigenvectors can be very large, and it is often 
difficult to get good bounds on the spectrum. Nonetheless, in Appendices B-D, we study both the weak $(\sigma=\epsilon \rightarrow 0)$ and strong $\left(\sigma=\epsilon^{-1} \rightarrow \infty\right)$ coupling limits, and show the following properties: (i) the spectral gap is $O(\epsilon)$, in both the strong and weak coupling limits-for strong driving, the decrease of the gap for larger $\sigma$ is consistent with the general occurrence in open systems [46]; (ii) the eigenvectors satisfy $\left|R_{j}\right\rangle=\mathcal{S}\left|\Phi_{j}\right\rangle$ and $\left|L_{j}\right\rangle=\mathcal{S}^{\dagger,-1}\left|\Phi_{j}\right\rangle$, for some invertible matrix $\mathcal{S}$ and an orthonormal basis $\left|\Phi_{j}\right\rangle$. The condition number of $\mathcal{S}$ is $\kappa(\mathcal{S}) \equiv\|\mathcal{S}\|\left\|\mathcal{S}^{-1}\right\|$ and satisfies $\kappa(\mathcal{S})=O(1+\epsilon)$. Moreover, in Secs. III B and IV we discuss some cases where the Liouvillian gap is independent of $q$. Models whose mixing time is independent of $q$ have also been obtained in Ref. [34], at the expense of more stringent requirements on the fluctuating terms of the Hamiltonian.

We then get that

$$
\left\|e^{t \mathcal{L}^{q}}-\mathcal{U}_{\infty}\right\|_{2 \rightarrow 2} \leq e^{-t \lambda^{*}} d^{2 q} \kappa(\mathcal{S})^{2},
$$

where $\lambda^{*}$ is the eigenvalue with the smallest nonzero real part and $\kappa(\mathcal{S})=\mathcal{O}(1+\epsilon)$. In terms of the trace norm, we then get that

$$
\sup _{\rho}\left\|e^{t \mathcal{L}^{q}}(\rho)-\mathcal{U}_{\infty}(\rho)\right\| \leq e^{-t \lambda^{*}} d^{4 q} \kappa(\mathcal{S})^{2}
$$

In the weak or strong coupling limits, the condition number will be of order 1 , yielding a mixing time of $T^{*} \sim 4 q \log (d) / \lambda^{*}$. We lose a lot in two steps of the bound, both times involving a term of order $d^{2 q}$. In certain cases, this is overly pessimistic. For instance, for a tensor product of $n$ semigroups, the mixing time is $T^{*} \sim \log (n) T_{1}^{*}$, where $T_{1}^{*}$ is the mixing time of a single subsystem [47]. We might ask whether the mixing time of Eq. (7) is also of the order $T^{*} \sim \log (q) T_{1}^{*}$, with $T_{1}^{*}=O\left(1 / \lambda^{*}\right)$.

We can see that this is not the case from the following argument,

$$
\begin{aligned}
\left\|e^{t \mathcal{L}^{q}}-\mathcal{U}_{\infty}\right\|_{1 \rightarrow 1} & \geq\left\|e^{t \mathcal{L}^{q}}-\mathcal{U}_{\infty}\right\|_{2 \rightarrow 2} \\
& \geq \sum_{j: \operatorname{Re}\left[\lambda_{j}\right]=-\lambda^{*}} e^{t \operatorname{Re}\left[\lambda_{j}\right]},
\end{aligned}
$$

since the lower bound is saturated when $\mathcal{S}=\mathbb{1}$, and we have isolated the subspace with eigenvalue $\lambda^{*}$. Now, in Sec. II C we argue that if the gap of $\mathcal{L}^{q}$ is the same as the gap of $\mathcal{L}^{1}$, then we can construct the eigenvectors with minimal nonzero eigenvalue of $\mathcal{L}^{q}$ from those of $\mathcal{L}^{1}$. In particular, the size of this subspace is at least as large as the size of the ground-state subspace. But we know that the ground-state subspace has dimension $d_{0} \geq e^{O(q)}$. Hence, the first excited subspace does as well. Then,

$$
\left\|e^{t \mathcal{L}^{q}}-\mathcal{U}_{\infty}\right\|_{1 \rightarrow 1} \geq e^{O(q)} e^{-t \lambda^{*}} .
$$

Thus, the mixing time is at least $T^{*} \sim O\left(q / \lambda^{*}\right)$, even in the weak coupling limit.

Finally, we comment on the distinction between the singular value gap of $e^{t \mathcal{L}}$ and the eigenvalue gap of $\mathcal{L}$. We know that as $t \rightarrow \infty$, the singular value gap $s^{*}(t)$, namely, the largest singular value $s_{j}(t) \neq 1$, converges to $e^{t \lambda^{*}}$; however, it is not clear how rapidly this occurs. This is discussed in the numerical studies of Sec. IV, where we show that, in both the strong and weak coupling limits, the difference between the spectral gap and the singular value gap vanishes on a time scale much smaller than $1 / \lambda^{*}$.

\section{MANY-BODY THEORY OF UNITARY DESIGN}

In the previous section we argue that bounding the spectral gap of the dynamical semigroup is in many relevant cases sufficient to get good estimates on the mixing time of the process. Here, we study such a gap by introducing a general mapping from a control Liouvillian to a non-Hermitian many-body Hamiltonian, and then study its mean-field solution. The mean-field approach has already been successfully applied [15] to estimate the convergence time of permutationally invariant random quantum circuits, where at each step a gate from a universal set is applied to a random pair of qubits. Moreover, in Sec. IV we analyze an integrable example via Bethe-ansatz techniques, from whose solution it appears that the eigenstates with the smallest gap are constructed from the steady states by changing the internal state of a single unpaired particle. This fact shares several similarities with what happens in bosonic condensates, and in particular with their mean-field solution [48]. Motivated by these two examples, it is natural to apply the mean-field analysis to generic Hamiltonian evolutions with random pulses. However, although the predictions of the mean-field solution are consistent with several numerical simulations, we clarify that this approach cannot be general by constructing explicit counterexamples via symmetry-breaking arguments.

\section{A. Mapping to a non-Hermitian many-body Hamiltonian}

A powerful method for estimating the spectral gap of the Liouvillian is to map Eq. (8) to a many-body problem, and then use powerful techniques developed in condensed-matter systems to obtain the spectrum. In order to find this mapping, we introduce a basis $b_{\alpha \beta}=|\alpha\rangle\langle\beta|, \alpha, \beta=1, \ldots, d$ and call $B_{\alpha \beta}=b_{\alpha \beta}^{\oplus q}$. These operators satisfy the $\mathrm{SU}(d)$ commutation relation, $\left[B_{\alpha \beta}, B_{\gamma \delta}\right]=B_{\alpha \delta} \delta_{\beta \gamma}-\delta_{\alpha \delta} B_{\beta \gamma}$, and therefore define a reducible representation of $\mathrm{SU}(d)$. Moreover, $\stackrel{\circ}{ }^{\oplus q}=$ $X^{\oplus q} \otimes \mathbb{1}-\mathbb{1} \otimes X^{\oplus q T}=\sum_{\alpha \beta}\left(X_{\alpha \beta} B_{\alpha \beta}^{\uparrow}-\left(X^{T}\right)_{\alpha \beta} B_{\alpha \beta}^{\downarrow}\right)$, where 
we set $B_{\alpha \beta}^{\uparrow}=B_{\alpha \beta} \otimes \mathbb{1}$ and $B_{\alpha \beta}^{\downarrow}=\mathbb{1} \otimes B_{\alpha \beta}$. Hence, the Liouvillian can be written as

$$
\begin{aligned}
\mathcal{L}_{q}= & -i \sum_{\alpha \beta} H_{\alpha \beta}\left(B_{\alpha \beta}^{\uparrow}-B_{\beta \alpha}^{\downarrow}\right) \\
& -\frac{\sigma}{2} \sum_{\alpha \beta \gamma \delta} V_{\alpha \beta} V_{\gamma \delta}\left(B_{\alpha \beta}^{\uparrow}-B_{\beta \alpha}^{\downarrow}\right)\left(B_{\gamma \delta}^{\uparrow}-B_{\delta \gamma}^{\downarrow}\right) .
\end{aligned}
$$

The form Eq. (24) is a convenient starting point because it depends only on the original $d \times d$ operators introduced in Eq. (1), while the complicated action into the $q$-copy Hilbert space is transferred into the basis operators $B$.

The operators $B$ form a reducible representation of $\mathrm{SU}(d)$ and can be decomposed in terms of irreducible operators that act on different invariant subspaces of the original $\left(\mathbb{C}^{d}\right)^{\otimes q}$ Hilbert space. Indeed, because of the Schur-Weyl duality, every irreducible representation of $\left(\mathbb{C}^{d}\right)^{\otimes q}$ is decomposed as $\left(\mathbb{C}^{d}\right)^{\otimes q}=\bigotimes_{\lambda} \mathcal{P}^{\lambda} \otimes U^{\lambda}$, where $\mathcal{P}^{\lambda}$ is an irreducible representation of the symmetric group $S_{q}$ and $\mathcal{U}^{\lambda}$ an irreducible representation of $\mathrm{SU}(d)$. A convenient expression for the fully symmetric and fully antisymmetric subspaces is given by [49] $B_{\alpha \beta}=a_{\alpha}^{\dagger} a_{\beta}$, where $a_{\alpha}$ and $a_{\alpha}^{\dagger}$ are either bosonic or fermionic creation and annihilation operators. Moreover, even a generic (though reducible) representation can be constructed from either bosonic or fermionic annihilation operators by adding an extra index and writing $B_{\alpha \beta}=\sum_{u} a_{\alpha u}^{\dagger} a_{\beta u}$. From the definition of $B$ one realizes that in this generic representation there are exactly $q$ particles since

$$
\sum_{\alpha u} a_{\alpha u}^{\dagger} a_{\alpha u}=\sum_{\alpha} B_{\alpha \alpha}=q \rrbracket .
$$

For convenience, we also perform the calculation in the basis where $V$ is diagonal. Therefore, Eq. (24) becomes

$$
\begin{aligned}
\mathcal{L}_{q}= & -i \sum_{\alpha \beta u} H_{\alpha \beta}\left(a_{\alpha u \uparrow}^{\dagger} a_{\beta u \uparrow}-a_{\beta u \downarrow}^{\dagger} a_{\alpha u \downarrow}\right) \\
& -\frac{\sigma}{2} \sum_{\alpha \beta u v} V_{\alpha \alpha} V_{\beta \beta}\left(n_{\alpha u \uparrow}-n_{\alpha u \downarrow}\right)\left(n_{\beta v \uparrow}-n_{\beta v \downarrow}\right),
\end{aligned}
$$

where $n_{x}=a_{x}^{\dagger} a_{x}$. Thanks to this general representation, the many-body Liouvillian has been mapped to a many-particle Hubbard-like problem Eq. (27) where the hopping part is anti-Hermitian. The original dependence on $q$ is mapped to the number of particles, namely to the constraint Eq. (25) that there are exactly $q$ particles in the "spin-up" and "spin-

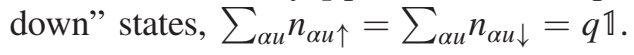

\section{B. Mean-field approach}

We consider here the decomposition Eq. (12) where each $\mathcal{L}_{k}^{\text {loc }}$ for different $k$ is equivalent to a single-copy Liouvillian
$\mathcal{L}_{1}$. From the above decomposition it is clear that if the gap of $\mathcal{L}^{\text {loc }}=\sum_{k} \mathcal{L}_{k}^{\text {loc }}$ equals the gap of $\mathcal{L}_{q}$, then the Liouvillian gap $\lambda^{*}$ is independent of $q$.

Extending the treatment of Sec. III A, we define a local basis of operators $\tilde{B}_{\tilde{\alpha} \tilde{\beta}}=B_{\alpha_{\uparrow} \beta_{\uparrow}}^{\uparrow} \delta_{\alpha_{\downarrow} \beta_{\downarrow}}+\delta_{\alpha_{\uparrow} \beta_{\uparrow}} B_{\alpha_{\downarrow} \beta_{\downarrow}}^{\downarrow}$, where $\tilde{\alpha}=\left(\alpha_{\uparrow}, \alpha_{\downarrow}\right)$, and similarly for $\tilde{\beta}$, are multi indices running from 1 to $d^{2}$. Therefore, we can write the decomposition Eq. (12) as

$$
\mathcal{L}_{q}=\sum_{\tilde{\alpha}, \tilde{\beta}}\left(\mathcal{L}_{1}^{\text {loc }}\right)_{\tilde{\alpha} \tilde{\beta}} \tilde{B}_{\tilde{\alpha} \tilde{\beta}}-\frac{\sigma}{2} \sum_{\tilde{\alpha}, \tilde{\beta}, \tilde{\gamma}, \tilde{\delta}} \stackrel{\circ}{V}_{\tilde{\alpha} \tilde{\beta}} \stackrel{\circ}{\tilde{\gamma}}_{\tilde{\gamma} \tilde{\delta}}\left(\tilde{B}_{\tilde{\alpha} \tilde{\beta}} \tilde{B}_{\tilde{\gamma} \tilde{\delta}}-\tilde{B}_{\tilde{\alpha} \tilde{\delta}} \tilde{\delta}_{\tilde{\beta} \tilde{\gamma}}\right),
$$

and, writing $\tilde{B}_{\tilde{\alpha} \tilde{\beta}}=a_{\tilde{\alpha}}^{\dagger} a_{\tilde{\beta}}$ with bosonic operators, then

$$
\mathcal{L}_{q}=\sum_{\tilde{\alpha}, \tilde{\beta}}\left(\mathcal{L}_{1}^{\text {loc }}\right)_{\tilde{\alpha} \tilde{\beta}} a_{\tilde{\alpha}}^{\dagger} a_{\tilde{\beta}}-\frac{\sigma}{2} \sum_{\tilde{\alpha}, \tilde{\beta}, \tilde{\gamma}, \tilde{\delta}} \stackrel{\circ}{V}_{\tilde{\alpha} \tilde{\beta}} \mathscr{o}_{\tilde{\gamma} \tilde{\delta}} a_{\tilde{\alpha}}^{\dagger} a_{\tilde{\gamma}}^{\dagger} a_{\tilde{\beta}} a_{\tilde{\delta}} .
$$

We assume that $\mathcal{L}_{q}^{\text {loc }}$ is diagonalizable (with left and right eigenvectors) as $\left(\mathcal{L}_{q}^{\text {loc }}\right)_{\tilde{\alpha} \tilde{\beta}}=\sum_{j} Z_{\tilde{\alpha} j} \lambda_{j} Z_{j \tilde{\beta}}^{-1}$ for a nonsingular matrix $Z$, where $j=0$ corresponds to the steady state. Then we define new bosonic operators via the nonunitary Bogoliubov transformation $\quad \tilde{a}_{i}^{\prime}=\sum_{\tilde{\alpha}} Z_{\tilde{\alpha} i} a_{\tilde{\alpha}}^{\dagger}, \quad \tilde{a}_{i}=$ $\sum_{\tilde{\alpha}}\left(Z^{-1}\right)_{i \tilde{\alpha}} a_{\tilde{\alpha}}$. These operators still satisfy the canonical commutation relations $\left[\tilde{a}_{i}, \tilde{a}_{j}{ }^{\prime}\right]=\tilde{\delta}_{i j}$, though $\tilde{a}_{i}{ }^{\prime} \neq \tilde{a}_{i}^{\dagger}$. As we show in Appendix G, in this language, the steady state of the many-body Liouvillian Eq. (28) is therefore the boson "condensate" $|\Omega\rangle=\left[\left(\tilde{a}_{0}^{\prime}\right)^{q} / \sqrt{q} !\right]|0\rangle$, where $|0\rangle$ is the bosonic vacuum. Elementary excitations with respect to this state can be constructed with a Bogoliubov (mean-field) approach by defining a variational wave function $|\psi\rangle=\sum_{j} \psi_{j}\left[\left(\tilde{a}_{0}{ }^{\prime}\right)^{q-1} / \sqrt{(q-1) !}\right] a_{j}|0\rangle$, for $j \neq 0$ and optimizing over the amplitudes $\psi_{j}$. These states are motivated by the analytic solution of the integrable model considered in Sec. IV, where the excited states with minimal gap have a single quasiparticle excitation. Although mean-field techniques have been highly studied mostly for Hamiltonian systems [48], they can also be extended to non-normal operators [50] where left and right eigenvectors form a biorthonormal basis. Within this variational formalism, we show in Appendix $G$ that the four-body interaction in Eq. (28) does not alter the eigenstates, which are therefore exactly given by the bare single-particle eigenstates $\left|\Omega_{j}^{\text {exc }}\right\rangle=\left[\left(\tilde{a}_{0}^{\prime}\right)^{q-1} / \sqrt{(q-1) !}\right] a_{j}|0\rangle$ with exact eigenvalue $\lambda_{j}$, for any $q$. This shows that the eigenvalues, at least in the low-energy subspace, are not "renormalized" for larger values of $q$. The obtained states $\left|\Omega_{j}^{\text {exc }}\right\rangle$ are indeed the symmetric combination of Eq. (15), which, as shown before, are an exact eigenstate of $\mathcal{L}_{q}$. Within this simple mean-field treatment there are no other eigenvalues with a smaller gap than $\min _{j}\left|\operatorname{Re}\left[\lambda_{j}\right]\right|$. Therefore, the final outcome of the 
mean-field treatment is that, at least for fully symmetric states, the Liouvillian gap is constant as a function of $q$.

\section{Counterexample to the mean-field treatment}

The mean-field treatment of the previous section, based on single-particle excitations, predicts that the Liovillian gap is independent of $q$, as long as the mean-field approach is accurate. Also, the rigorous Bethe-ansatz treatment of Sec. IV, valid for a particular integrable model, shows that the Liouvillian gap is independent of $q$, by explicitly showing that the states with minimal gap are made by unpaired particles. That rigorous treatment thus justifies the mean-field approach, at least for that particular model. However, here we show that the predictions of the meanfield theory cannot be general by finding a counterexample where a state with two bounded particles (hence, appearing for $q \geq 2$ ) may have a lower gap.

We construct this counterexample via symmetry arguments. Clearly, in the fully controllable case $H$ and $V$ must not share a symmetry-otherwise only symmetric unitaries can be obtained-but this lack of common symmetries is not sufficient. Indeed, generically, in tensor copies there may be other nontrivial symmetries but, because of the Schur-Weyl duality, in the fully controllable case only the permutation symmetries can remain. Suppose now that our system is not controllable because there exists an operator $\tilde{X}$, different from a permutation operator, such that $\left[H^{\oplus p}, \tilde{X}\right]=\left[V^{\oplus p}, \tilde{X}\right]=0$ and that the solutions of $\left[H^{\oplus q}, X\right]=\left[V^{\oplus q}, X\right]=0$ for $q<p$ are only permutation operators. In this case, Eq. (11) would be valid for $q<p$, but not when $p=q$, as the symmetry $\tilde{X}$ introduces an extra steady state. Then, suppose that we restore full controllability by adding a small $\mathcal{O}(\epsilon)$ term in either $H$ or $V$ such that the operator $\tilde{X}$ is not a symmetry anymore (we say that the symmetry $\tilde{X}$ is explicitly broken). This splits the extra steady state into an eigenvector with small $\mathcal{O}(\epsilon)$ eigenvalue, which, for small enough $\epsilon$, can be smaller than the gap, obtained when $q<p$. If this counterexample can be constructed, then the gap for $q<p$ may be different from the gap at $q=p$. Below, we show that this construction is indeed possible already with $p=2$ and that these extra eigenstates correspond to bound particles in the many-body framework.

As shown in Refs. [51,52], a rather surprising necessary and sufficient condition for controllability is that there are exactly two independent solutions of the equations $\left[H^{\oplus 2}, X\right]=\left[V^{\oplus 2}, X\right]=0$. Nonetheless, a simpler necessary condition (though not sufficient [51]) is the absence of nonzero solutions to the set of equations

$$
Q H^{T}+H Q=Q V^{T}+V Q=0 .
$$

Taking the complex conjugate of Eq. (29), we find that $Q$ satisfies $Q^{*} H+H^{T} Q^{*}=Q^{*} V+V^{T} Q^{*}=0$, as $H$ and $V$ are Hermitian. Because of this, $Q Q^{*}$ commutes with both $H$ and $V$ and, owing to Schur's lemma, $Q Q^{*}$ is proportional to the identity. Reference [53] proved that $Q Q^{*}=1$ when $Q$ is symmetric and $Q Q^{*}=-1$ when $Q$ is antisymmetric. If there are nonzero solutions of Eq. (29), then the system is not controllable and there are extra steady states such as the bosonic paired state for $q=2$ :

$$
\left|\psi_{Q}\right\rangle=\sum_{\tilde{\alpha} \tilde{\beta}}\left(Q \otimes Q^{*}\right)_{\tilde{\alpha} \tilde{\beta}} a_{\tilde{\alpha}}^{\dagger} a_{\tilde{\beta}}^{\dagger}|0\rangle .
$$

Indeed, for both $Q$ symmetric and antisymmetric, $Q \otimes Q^{*}$ is symmetric, thus justifying the bosonic approach. The proof can be readily obtained from Eq. (28), indeed for both $X=H, V$ :

$$
\begin{aligned}
\stackrel{X}{X} \tilde{\gamma} \tilde{\delta}_{\tilde{\gamma}}^{\dagger} a_{\tilde{\delta}}\left|\psi_{Q}\right\rangle= & \stackrel{X}{\tilde{\gamma} \tilde{\delta}}_{\tilde{\delta}} a_{\tilde{\gamma}}^{\dagger} a_{\tilde{\delta}} \sum_{\tilde{\alpha} \tilde{\beta}} Q_{\tilde{\alpha} \tilde{\beta}} a_{\tilde{\alpha}}^{\dagger} a_{\tilde{\beta}}^{\dagger}|0\rangle \\
= & {\left[(X Q) \otimes Q^{*}+\left(Q X^{T}\right) \otimes Q^{*}+\right.} \\
& \left.-Q \otimes\left(X^{T} Q^{*}\right)-Q \otimes\left(Q^{*} X\right)\right]_{\tilde{\gamma} \tilde{\delta}} a_{\tilde{\gamma}}^{\dagger} a_{\tilde{\delta}}^{\dagger}|0\rangle,
\end{aligned}
$$

so because of Eq. (29), we find $\stackrel{\circ}{H}_{\tilde{\gamma} \tilde{\delta}} a_{\tilde{\gamma}}^{\dagger} a_{\tilde{\delta}}\left|\psi_{Q}\right\rangle=$ $\stackrel{\circ}{V}_{\tilde{\gamma} \tilde{\delta}} a_{\tilde{\gamma}}^{\dagger} a_{\tilde{\delta}}\left|\psi_{Q}\right\rangle=0$, namely, $\mathcal{L}_{2}\left|\psi_{Q}\right\rangle=0$. Hence, the extra symmetry $Q$ introduces a pairing between bosons in the steady state, which is expressed by Eq. (30) —note that it is indeed a pairing because $\left[Q \otimes Q^{*}\right]_{\tilde{\alpha} \tilde{\beta}} \neq Q_{\tilde{\alpha}} Q_{\tilde{\beta}}^{*}$ since $Q$ is a matrix.

As discussed before, we can restore controllability by explicitly breaking the symmetry Eq. (29) with small terms: $Q H^{T}+H Q=\epsilon_{H}, Q V^{T}+V Q=\epsilon_{V}$, where at least one between $\epsilon_{V}$ or $\epsilon_{H}$ has to be nonzero, otherwise the system is not controllable. In this case, $\left|\psi_{Q}\right\rangle$ is not a steady state but, within first-order perturbation theory, can be used to create a state with eigenvalue $\tilde{\delta}=O\left(\epsilon_{V}, \epsilon_{H}\right)$. In particular, one can construct specific examples where $\epsilon_{V}$ and $\epsilon_{H}$ are much smaller than the gap $\lambda^{*}$ of $\mathcal{L}_{1}$ so that $\tilde{\delta}<\lambda^{*}$. Therefore, exploiting these broken symmetries we can construct counterexamples where the gap changes as a function of $q$. The simplest example is a two-spin system with $H=$ $\left(\sigma_{1}^{x} \sigma_{2}^{x}+\sigma_{1}^{y} \sigma_{2}^{y}+\sigma_{1}^{x}\right)+\epsilon \sigma_{1}^{z} \sigma_{2}^{z}$ and $V=\sigma_{1}^{y}$, where $\sigma_{j}^{\alpha}$ are the Pauli matrices acting on the spin $j$. For instance, for $\epsilon=0.1$, the gap of $\mathcal{L}_{1}$ is $\approx 0.45$, while the gap of $\mathcal{L}_{2}$ is $\approx 0.05$.

In spite of this counterexample, we observe that in most numerical examples, performed for small values of $d$ and $q$ with a random choice of $H$ and $V$, the Liouvillian gap is constant as a function of $q$. This allows us to conjecture that "typically," namely for most choices of $H$ and $V$, the Liouvillian has a constant gap, as predicted by the mean-field approach. Since in Eq. (12) each copy interacts with all the others, this 
conjecture is supported by the well-known validity (see, e.g., Ref. [42]) of the mean-field solution in long-range models.

\section{CONTROLLABLE QUANTUM WALK}

We focus on a specific model that is of experimental interest, namely, a single-particle hopping in a one-dimensional lattice; see Fig. 1. This framework can describe different physical systems, such as a spin impurity in a spin chain, a single electronic excitation in quantum dot arrays, and a photon traveling in a one-dimensional photonic chip. The resulting quantum walk can be modeled via the Hamiltonian

$$
H=\sum_{n=1}^{L-1}|n\rangle\langle n+1|+\text { H.c. },
$$

where $|n\rangle$ represents the state in which the walker is in position $n$ and $L$ is the length of the chain. This Hamiltonian has found numerous applications in quantum transport problems and remote entanglement generation in spin chains [54-57].

Moreover, we consider a local control field on a single site of the chain, namely the $c$ th site, which is modeled by the Hamiltonian term $g(t) V$, where $V=|c\rangle\langle c|$ and $g(t)$ is a time-dependent control profile. One can show that the chain is controllable provided that $c$ and $L+1$ are co-prime numbers $[58,59]$. For simplicity, in the following we set $c=1$. The above hopping Hamiltonian with local control can be realized in many physical systems, for example, in reconfigurable photonic chips $[21,22]$, where the different control pulses can be obtained by electrically tuned on-chip heaters [23].

In the following we evaluate the Liouvillian gap for all possible values of $q$ in the strong-driving limit, namely, when $\sigma \gg 1$. The opposite weak-driving limit is discussed

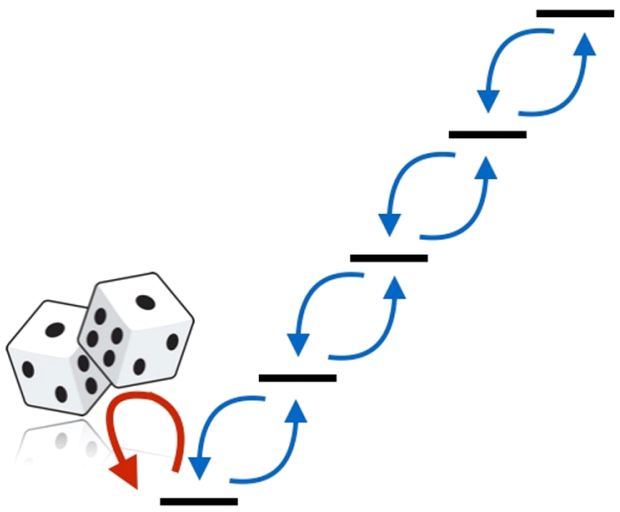

FIG. 1. Depiction of a one-dimensional quantum walk, with a local control at the bottom rung. Each site of the chain is coherently coupled with its nearest neighbors. Random control pulses are applied to the first site. in Appendix C for the single-particle $q=1$ case. We start by considering two important cases, namely, the fully symmetric and fully antisymmetric representation where $B_{\alpha \beta}=a_{\alpha}^{\dagger} a_{\beta}$ for either bosonic or fermionic d.o.f. We then extend our analysis to the general case.

\section{A. Gap analysis: Fully symmetric representation}

We consider first the fully symmetric representation where $B_{\alpha \beta}=a_{\alpha}^{\dagger} a_{\beta}$, so one can omit the index $u$ from the equations of Sec. III A. Plugging the operators $H$ and $V$ of the controllable chain into Eq. (27), one finds the following Liouvillian:

$$
\begin{aligned}
\mathcal{L}_{q}= & -i \sum_{\alpha}\left(a_{\alpha \uparrow}^{\dagger} a_{\alpha+1, \uparrow}-a_{\alpha \downarrow}^{\dagger} a_{\alpha+1, \downarrow}+\text { H.c. }\right) \\
& -\frac{\sigma}{2}\left(n_{1}^{\uparrow}-n_{1}^{\downarrow}\right)\left(n_{1}^{\uparrow}-n_{1}^{\downarrow}\right) .
\end{aligned}
$$

To diagonalize the above operator we assume that $\sigma \gg 1$ and we study the "low-energy" effective dynamics. In that limit the dissipative part $\sigma \mathcal{D}=(\sigma / 2)\left(n_{1}^{\uparrow}-n_{1}^{\downarrow}\right)\left(n_{1}^{\uparrow}-n_{1}^{\downarrow}\right)$ has either eigenvalue 0 or $\sigma \gg 1$. With a perturbative approach, discussed in Appendix D, we decouple the latter "high-energy" subspace and obtain an effective Liouvillian acting in the low-energy sector. From a first-order expansion as a function of $\sigma^{-1}$ the effective Liouvillian is given by

$$
\begin{aligned}
\hat{\mathcal{L}_{q}}= & \frac{2}{\sigma}-\frac{2}{\sigma} \sum_{k=1}^{L-1} g_{k}\left[-2\left(\tilde{a}_{0 \uparrow}^{\dagger} \tilde{a}_{0 \downarrow}^{\dagger} \tilde{a}_{k \uparrow} \tilde{a}_{k \downarrow}+\text { H.c. }\right)\right. \\
& \left.+\left(\tilde{n}_{0 \uparrow}+\tilde{n}_{0 \downarrow}+1\right)\left(\tilde{n}_{k \uparrow}+\tilde{n}_{k \downarrow}+1\right)\right],
\end{aligned}
$$

where $g_{k}=(2 / L) \sin ^{2}(\pi k / L), \quad \tilde{a}_{k \uparrow}=\sum_{\alpha=1}^{L-1}(2 / L) \sin ^{2} \times$ $(\pi k \alpha / L) a_{\alpha+1, \uparrow}, \quad$ and $\quad a_{1 \uparrow} \equiv \tilde{a}_{0 \uparrow}$. We now call $K_{i}^{+}=\tilde{a}_{i \uparrow}^{\dagger} \tilde{a}_{i \downarrow}^{\dagger}, K_{i}^{-}=\left(K_{i}^{+}\right)^{\dagger}$, and $K_{i}^{z}=\left(\tilde{n}_{i \uparrow}+\tilde{n}_{i \downarrow}+1\right) / 2$ and note that these operators satisfy the $\mathrm{SU}(1,1)$ commutation relations:

$$
\begin{gathered}
{\left[K_{i}^{+}, K_{i}^{-}\right]=-2 K_{i}^{z}, \quad\left[K_{i}^{z}, K_{i}^{ \pm}\right]= \pm K_{i}^{ \pm},} \\
{\left[K_{i}^{\alpha}, K_{j}^{\beta}\right]=0, \quad \text { if } i \neq j .}
\end{gathered}
$$

With these definitions, we find then

$$
\hat{\mathcal{L}_{q}}=\frac{2}{\sigma}-\frac{8}{\sigma} \sum_{k=1}^{L-1} g_{k} K_{0} \cdot K_{k},
$$

where $K_{i} \cdot K_{j} \equiv-\left(K_{i}^{+} K_{j}^{-}+K_{i}^{-} K_{j}^{+}\right) / 2+K_{i}^{z} K_{j}^{z}$ is the $\mathrm{SU}(1,1)$ invariant product, namely, the analogue of the Heisenberg interaction. The model Eq. (36) is a $\mathrm{SU}(1,1)$ Gaudin model [60], which is known to be exactly solvable with the Bethe-ansatz approach. We explicitly diagonalize 
it in Appendix E by applying Richardson's method [61]. We find that the eigenvalues of the Liouvillian $\hat{\mathcal{L}_{q}}$ are

$$
\lambda=-\frac{2}{\sigma}\left(\sum_{k} g_{k} n_{k}+4 \sum_{\alpha} E_{\alpha}\right)
$$

where the non-negative integers $n_{k}$ parametrize the number of unpaired particles in mode $k$ (see the discussion in Appendix E) and the $E_{\alpha}$ are either zero or the solution of the nonlinear set of equations

$$
\sum_{k} \frac{n_{k}+1}{\omega_{\alpha}-2 g_{k}^{-1}}+\frac{1}{\omega_{k}}+2 \sum_{\beta \neq \alpha} \frac{1}{\omega_{\alpha}-\omega_{\beta}}=0,
$$

where $E_{\alpha}=1 / \omega_{\alpha}$. From that expression it is clear that the steady state corresponds to $E_{\alpha}=0$ and $n_{k}=0$, for each $\alpha$ and $k$. Solutions to the above equations are known to be related with the roots of Heine-Stieltjes polynomials (see, e.g., Ref. [62]). By exploiting this relationship, one finds that all the solutions $\omega_{\alpha}$ of Eq. (38) are real, different from each other, and different from the poles of Eq. (38). Moreover, $g_{k}=g_{L-k}$ so the sum in Eq. (38) can be restricted to the first half where $g_{k}<g_{k+1}$. The roots of the Heine-Stieltjes polynomials also have the important property that they lie inside the intervals $2 g_{k+1}^{-1}<\omega_{\alpha}<$ $2 g_{k}^{-1}$ for some $k$, so that $2 E_{\alpha}>\min _{k} g_{k}=g_{1}$. This constraint allows us to rigorously find the gap of the Liouvillian $\hat{\mathcal{L}_{q}}$. Indeed, thanks to the latter inequality, the paired states have a larger gap than the unpaired ones, so we can focus only on the solutions where $E_{\alpha}=0$. The minimum gap is then obtained when $n_{1}=n_{L-1}=1$ and $n_{k}=0$ otherwise. This is an allowed state (for $L>2$ ) as it satisfies all the constraints and provides the gap

$$
\text { gap } \equiv \lambda^{*}=\frac{8}{\sigma L} \sin ^{2}\left(\frac{\pi}{L}\right)=O\left(L^{-3}\right) .
$$

This gap is exact in the strong-driving limit, can be achieved already at $q=1$, and is the same for all higher values of $q$, as we have shown that there are no smaller nonzero eigenvalues. Therefore, we prove here explicitly that in the strong-driving limit the gap is independent of the number of copies $q$. In the following sections we extend this result, which up to now is restricted to the fully symmetric representation, to show that Eq. (39) is indeed the gap, irrespective of the chosen representation.

\section{B. Gap analysis: Antisymmetric representations}

We first consider another particular case, namely, the fully antisymmetric representation, that is used as a basis for the general solution discussed in the next section. We start from Eq. (27) and we write $B_{i j}^{\alpha}=a_{i \alpha}^{\dagger} a_{j \alpha}$, with fermionic creation and annihilation operators. Repeating the effective Liouvillian description of the previous section, we find

$$
\hat{\mathcal{L}}_{q}=-\frac{2}{\sigma}+\frac{8}{\sigma} \sum_{k=1}^{L-1} g_{k} S_{0} \cdot S_{k}
$$

where $S_{0} \cdot S_{k}=\sum_{\alpha=x, y, z} S_{0}^{\alpha} S_{k}^{\alpha}$ refers to the $\mathrm{SU}(2)$-invariant product, namely, the spin Heisenberg interaction $S_{j}^{ \pm}=S_{j}^{x} \pm i S_{j}^{y}$, and where we define $S_{j}^{-}=\tilde{a}_{j \uparrow} \tilde{a}_{j \downarrow}, S_{j}^{+}=$ $\left(S_{j}^{-}\right)^{\dagger}$ and $S_{j}^{z}=\left(\tilde{a}_{j \uparrow}^{\dagger} \tilde{a}_{j \uparrow}+\tilde{a}_{j \downarrow}^{\dagger} \tilde{a}_{j \downarrow}-1\right) / 2$. It is simple to verify that the above operators satisfy the $\mathrm{SU}(2)$ commutation relations on the same site, and commute on different sites, so that Eq. (40) is equivalent to the central spin model first studied by Gaudin [60]. The diagonalization of the Gaudin Heisenberg Hamiltonian proceeds along the same lines of the $\mathrm{SU}(1,1)$ one. There are two main differences: (i) the different sign in Eqs. (40) and (36) and (ii) because of the Pauli exclusion principle the number of particles $n_{k}$ per mode $k$ is limited to either 0 or 1 . We find then that the eigenvalues are given by Eq. (37), where the nonzero energies $E_{\alpha}$ are the solutions of

$$
\sum_{k} \frac{g_{k}\left(n_{k}-1\right)}{2 E_{\alpha}-g_{k}}-2 \sum_{\beta \neq \alpha} \frac{E_{\beta}}{E_{\alpha}-E_{\beta}}=1 .
$$

However, because of the different sign in Eq. (41), we cannot relate the solutions of Eq. (41) to the roots of the Heine-Stieltjes polynomials, so we cannot bound the gap using the argument of the fully symmetric case. Nonetheless, in the next section we consider a more general technique, valid for all the representations, where such a bound can be obtained using physical arguments borrowed from classical electrostatics.

\section{General gap analysis}

As we discuss in Sec. III A, a general representation of the $\mathrm{SU}(L)$ algebra can can be obtained via extended creation and annihilation operators [49], namely, $B_{\alpha \beta}=$ $\sum_{u} \tilde{a}_{\alpha u}^{\dagger} \tilde{a}_{\beta u}$ for either bosonic or fermionic operators. We use the fermionic representation for convenience, since our derivation uses the particle-hole symmetry that is a nonunitary operation in bosonic systems (see, e.g., Ref. [48]). Because of the Pauli exclusion principle, in order to satisfy the constrain $\sum_{\alpha} B_{\alpha \alpha}=q$, the auxiliary index $u$ has to run from 1 to $q$. Performing the same perturbative approach of Appendix D, valid in the strong-driving limit $\sigma \gg 1$, one finds that the effective Liouvillian $\hat{\mathcal{L}_{q}}$ can be written in the diagonal basis of the Hamiltonian as 


$$
\begin{aligned}
\hat{\mathcal{L}_{q}}= & -\frac{2}{\sigma} \sum_{k=1}^{L-1} g_{k}\left(B_{0 k}^{\uparrow} B_{k 0}^{\uparrow}+B_{k 0}^{\uparrow} B_{0 k}^{\uparrow}+B_{0 k}^{\downarrow} B_{k 0}^{\downarrow}+B_{k 0}^{\downarrow} B_{0 k}^{\downarrow}\right. \\
& \left.-2 B_{0 k}^{\uparrow} B_{0 k}^{\downarrow}-2 B_{k 0}^{\uparrow} B_{k 0}^{\downarrow}\right) .
\end{aligned}
$$

The above Hermitian operator corresponds to the purely dissipative Liouvillian,

$$
\hat{\mathcal{L}^{q}} \rho=-\frac{2}{\sigma} \sum_{k=1}^{L-1} g_{k}\left(\left[\tilde{V}_{k}^{\oplus q},\left[\tilde{V}_{k}^{\dagger \oplus q}, \rho\right]\right]+\left[\tilde{V}_{k}^{\dagger \oplus q},\left[\tilde{V}_{k}^{\oplus q}, \rho\right]\right]\right),
$$

where $\tilde{V}_{k}=\left|\omega_{k}\right\rangle\left\langle\omega_{0}\right|$, with $\left|\omega_{k}\right\rangle=\sum_{j=1}^{L-1}(2 / L) \sin (\pi j k / L)^{2}$ $|j+1\rangle$ and $\left|\omega_{0}\right\rangle=|1\rangle$. One can check that the operators $\tilde{V}_{k}$ and their Hermitian conjugate form a controllable set, so the steady state of the effective Liouvillian coincides with the original one. We now perform two transformations. The first one is the Jordan-Wigner transformation to obtain proper fermionic d.o.f., namely, where creation and annihilation operators with different indices $\uparrow$ and $\downarrow$ anticommute. The second one is a particle-hole transformation in the spin-down sector. These transformations are implemented together by defining $W=\prod_{j u} e^{i \tilde{a}_{j u \uparrow}^{\dagger} \tilde{a}_{j u \uparrow}}$ and setting $a_{j u \uparrow}=\tilde{a}_{j u \uparrow} \quad$ and $\quad a_{j u \downarrow}=W \tilde{a}_{j u \uparrow}^{\dagger}$. Equation (42) then becomes

$$
\begin{aligned}
\hat{\mathcal{L}_{q}} & =-\frac{2}{\sigma} \sum_{k=1}^{L-1} g_{k} \sum_{\alpha \beta}\left[a_{0 \alpha}^{\dagger} a_{k \alpha} a_{k \beta}^{\dagger} a_{0 \beta}+a_{k \alpha}^{\dagger} a_{0 \alpha} a_{0 \beta}^{\dagger} a_{k \beta}\right] \\
& =-\frac{2 q}{\sigma}+\frac{4}{\sigma} \sum_{k=1}^{L-1} g_{k} \sum_{\alpha \beta} X_{\alpha \beta}^{(0)} X_{\beta \alpha}^{(k)},
\end{aligned}
$$

where $X_{\alpha \beta}^{(j)}=\left(a_{j \alpha}^{\dagger} a_{j \beta}-a_{j \beta} a_{j \alpha}^{\dagger}\right) / 2$ and the Greek letters refer to the multi-index composed by the auxiliary index and the "effective spin" index, i.e., $\alpha=(u s)$, where $u=$ $1, \ldots, q$ and $s=\{\uparrow, \downarrow\}$. The traceless operators $X_{\alpha \beta}^{(j)}$ satisfy the $\mathrm{SU}(2 q)^{\oplus L}$ commutation relations,

$$
\left[X_{\alpha \beta}^{(j)}, X_{\gamma \delta}^{(k)}\right]=\delta_{j k}\left(X_{\alpha \delta}^{(j)} \delta_{\beta \gamma}-X_{\gamma \beta}^{(j)} \delta_{\alpha \delta}\right),
$$

so that Eq. (43) represents a $\mathrm{SU}(2 q)$ version of the Gaudin model. Indeed, Eq. (43) is invariant under the Bogoliubov transofmation $a_{j \alpha} \rightarrow \sum_{\beta} U_{\alpha, \beta} a_{j \beta}$, where $U$ is a unitary $(2 q) \times(2 q)$ matrix. $\mathrm{SU}(2 q)$ has $(2 q)^{2}-1$ generators, so one operator in Eq, (44) is dependent of the others. This is shown by the equation $\left[\sum_{\alpha} X_{\alpha \alpha}^{(j)}, X_{\beta \gamma}^{(k)}\right]=0$ for each $\beta$ and $\gamma$. Going back to the original representation, namely performing back the particle-hole transformation, one finds that

$$
X_{(x, \uparrow),(y, \uparrow)}^{(j)}=\frac{\tilde{a}_{j x \uparrow}^{\dagger} \tilde{a}_{j y \uparrow}-\tilde{a}_{j y \uparrow} \tilde{a}_{j x \uparrow}^{\dagger}}{2},
$$

$$
\begin{gathered}
X_{(x, \downarrow),(y, \downarrow)}^{(j)}=\frac{\tilde{a}_{j x \downarrow} \tilde{a}_{j y \downarrow}^{\dagger}-\tilde{a}_{j y \downarrow}^{\dagger} \tilde{a}_{j x \downarrow}}{2}, \\
X_{(x, \uparrow),(y, \downarrow)}^{(j)}=\tilde{a}_{j x \uparrow}^{\dagger} W \tilde{a}_{j y \downarrow}^{\dagger}, \\
X_{(x, \downarrow),(y, \uparrow)}^{(j)}=\tilde{a}_{j x \downarrow} W \tilde{a}_{j y \uparrow} .
\end{gathered}
$$

The Gaudin-like model Eq. (43) has been solved for different algebras [namely, not only the $\mathrm{SU}(1,1)$ and $\mathrm{SU}(2)$ cases discussed before] in Refs. $[63,64]$, while the duality between the different models that can be obtained by exploiting the auxiliary indices has different ramifications in mathematical physics (see, e.g., Ref. [65] and references therein), especially due to its connections with the Knizhnik-Zamolodchikov equation [65,66]. In Appendix F, we exploit the general solution $[63,64]$ of the Gaudin model Eq. (43), valid when the operators $X$ define any semisimple Lie algebra, to obtain the eigenvalues of the Liouvillian Eq. (43) when the $\mathrm{SU}(2 q)$ operators are defined via the fermionic representation Eq. (45). As in the fully symmetric and fully antisymmetric case discussed in the previous sections, the eigenvalues of $\hat{\mathcal{L}}_{q}$ are parametrized by non-negative integers $n_{\uparrow j}$ and $n_{\downarrow j}$, and are given by

$$
\lambda=-\frac{2}{\sigma}\left[\sum_{k=1}^{L-1} g_{k}\left(n_{\downarrow k}+n_{\uparrow k}\right)+4 \sum_{\alpha} \frac{1}{\omega_{q, \alpha}}\right],
$$

where $\omega_{j, \alpha}$ for $j=1, \ldots, 2 q-1$ are the solutions of

$$
\begin{aligned}
\sum_{\beta} \frac{2}{\omega_{j, \beta}-\omega_{j, \alpha}}= & \sum_{k=0}^{L-1} \frac{\mu_{j}^{k}}{z_{k}-\omega_{j, \alpha}}+\sum_{\beta} \frac{1}{\omega_{j+1, \beta}-\omega_{j, \alpha}} \\
& +\sum_{\beta} \frac{1}{\omega_{j-1, \beta}-\omega_{j, \alpha}},
\end{aligned}
$$

with $z_{0}=0, \mu_{j}^{0}=\delta_{q j}$, and, for $k>0, z_{k}=2 g_{k}^{-1}$ and $\mu_{j}^{k}=\delta_{j, q}\left(1-\delta_{n_{\downarrow k}>0}-\delta_{n_{\uparrow k}>0}\right)+\delta_{j, q+n_{\uparrow k}}+\delta_{j, q-n_{\downarrow k}}$. In Eq. (47) we set $\omega_{0, \beta}=\omega_{2 q, \beta} \rightarrow-\infty$; namely, in other words, for $j=1$ or $j=2 q-1$ one of the two fractions in the second line is zero.

Owing to the similarity between Eqs. (46) and (37), if we can show that the solutions of Eq. (47) satisfy the inequality $2 \omega_{q, \alpha}^{-1}>g_{k}$ for each $\alpha$ and $k$, then we can straightforwardly apply the reasoning of Sec. IVA to prove that the gap is indeed given by Eq. (39) for any representation. However, the sign difference between Eqs. (47) and (38) prevents us from using the theory of Heine-Stieltjes polynomials to prove that inequality, as we did in Sec. IVA. Here, we use a different approach, used also in Ref. [63] for a different purpose, which is based on mapping the mathematical equation (47) to an electrostatic problem, and then use our 
classical physics intuition. Following Ref. [63] we define the two-dimensional vector $\vec{\omega}_{j \alpha}$ whose real components are the real and imaginary part of $\omega_{j \alpha}$ and interpret those vectors as the positions of some particles with index $\alpha$ and species $j=1, \ldots, 2 q-1$. Equation (47) can then be interpreted as the conditions for an extremum of the function $\mathcal{W}(\{\omega\})$ defined as

$$
\begin{gathered}
\mathcal{W}(\{\omega\})=-\sum_{i, j=1}^{2 q-1} \sum_{\alpha \beta} C_{i j} \log \left|\vec{\omega}_{i \alpha}-\vec{\omega}_{j \beta}\right|-\sum_{i=1}^{2 q-1} \sum_{\alpha} \mathcal{V}_{i}\left(\vec{\omega}_{i \alpha}\right), \\
\mathcal{V}_{i}(\vec{\omega})=-\sum_{k=0}^{L-1} \mu_{i}^{k} \log \left|\vec{\omega}-\vec{z}_{k}\right|
\end{gathered}
$$

where $\vec{z}_{k}=\left(z_{k}, 0\right)$ and the Cartan matrix $C_{i j}$ has nonzero components only on the diagonal, where $C_{i i}=2$, and for $|i-j|=1$, where $C_{i j}=-1$. This shows that the problem of finding a solution to the system of equations (47) is equivalent to the problem of finding the equilibrium positions of a set of particles in a two-dimensional plane interacting via the logarithmic potential Eq. (48). That potential is analogous to the electrostatic potential since the Coulomb interaction in 2D is logarithmic. Particles of the same species repel each other, while particles with nearestneighbor species attract each other. Finding the equilibrium positions of those particles is in general quite complicated, although the problem can be solved explicitly in the thermodynamic limit [67]. At first sight one may think that the problem has no solutions since the potential Eq. (49) is unstable. However, because of the $Z_{2}$ symmetry $\left(\operatorname{Im}\left[\omega_{j, \alpha}\right] \rightarrow-\operatorname{Im}\left[\omega_{j, \alpha}\right]\right)$, due to the fact that the $z_{k}$ 's are real, all the forces on the real line are longitudinal. This property allows us to seek for solutions of Eq. (47) in the class of real numbers [63]. On the real line, the problem becomes stable and one dimensional. An example of this effective onedimensional potential is shown in Fig. 2 where one can see the two unbounded regions for $\omega<\min _{k} z_{k}$ and for

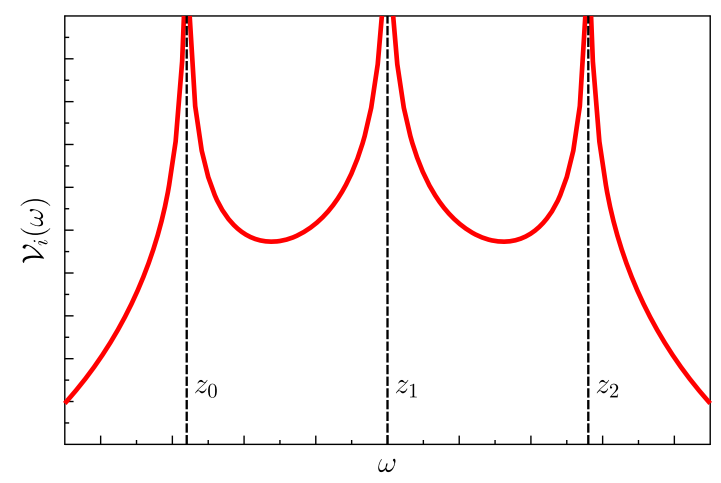

FIG. 2. Example one-dimensional potential $\mathcal{V}_{i}(\omega)$ from Eq. (49) with three different values of $z_{k}$ and $\mu_{i}^{k}=1$. $\omega>\max _{k} z_{k}$, where no solutions can exist. Therefore, this electrostatic analogy shows that the only stable solutions with finite $\omega_{i \alpha}$ can be found only between poles of $\mathcal{V}_{i}(\omega)$, or, in other words, that the solutions of the nonlinear set of equations (47) satisfy the constraint $\min _{k} z_{k}<$ $\omega_{j \alpha}<\max _{k} z_{k}$, i.e., $2 \omega_{j \alpha}^{-1}>\min _{k} g_{k}$. This, together with the discussion of Sec. IVA, shows that Eq. (39) is indeed the gap of the Liouvillian $\hat{\mathcal{L}}_{q}$ in the strong-driving limit.

\section{Numerical results for the controllable chain}

In the previous sections we perform an extensive theoretical analysis to show that, in a chain controlled on one boundary, the Liouvillian gap in the strong-driving limit is constant as a function of $q$ and scales as $\propto L^{-3}$ as a function of the length $L$ of the chain-this scaling is consistent with what has been obtained in spin chains with boundary dissipation [68]. The scaling $\propto L^{-3}$ is obtained also in the weak-driving limit discussed in Appendix C, though that analysis is valid only for $q=1$. Nontheless, in all our numerical experiments obtained for small values of $L$ and $q$, we find that the gap is constant as a function of $q$ over the whole range of $\sigma$. In Fig. 3, we study the Liouvillian gap and show that the theoretical predictions of the strong- and weak-driving limits are very accurate in their respective limit of validity. Moreover, we find that the accuracy of the strong-driving limit is not affected by the length of the chain. This is shown indeed in the inset Fig. 3, where one observes an almost constant behavior as a function of $L$. In Fig. 4, on the other hand, we show that the Liouvillian gap scales as $L^{-3}$ for different values of $\sigma$. This scaling has been predicted in the strong- and weakdriving limits by Eqs. (39) and (C12). However, Fig. 4 shows that such scaling is valid also for $\sigma \approx 2$ where neither

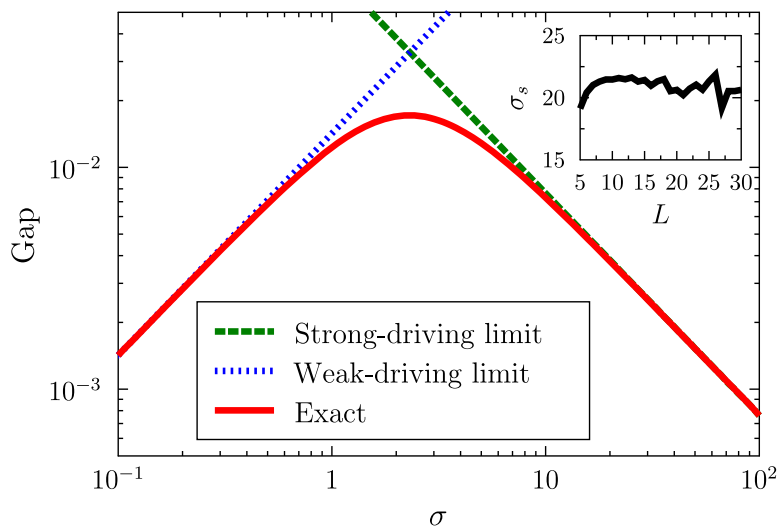

FIG. 3. Liouvillian gap for a controllable chain of $L=10$ as a function of the noise strength $\sigma$. Exact numerical results are obtained with $q=1$. Strong-driving limit corresponds to Eq. (39), while the weak-driving limit is from Eq. (C12). Inset: Noise strength $\sigma_{s}$ as a function of $L$ such that, for $\sigma>\sigma_{s}$, the relative error between the exact gap and the strong-coupling estimate is smaller than $1 \%$. 


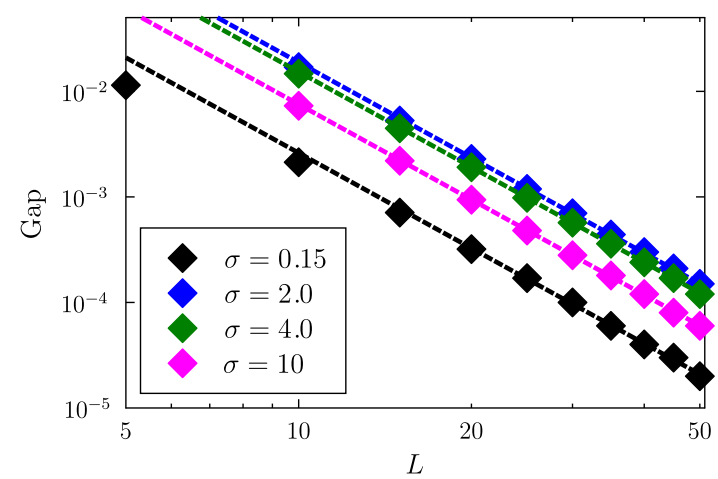

FIG. 4. Scaling of the Liouvillian gap obtained numerically for $q=1$ as a function of $L$ and for different values of $\sigma$. Solid lines correspond to fitting functions $\propto L^{-3}$.

the strong nor the weak coupling limit holds (compare, e.g., the values of Figs. 4 and 3). In Fig. 5, we study the relationship between the Liouvillian gap and the gap $s^{*}(t)$ in the singular values of $e^{t \mathcal{L}_{q}}$, which is a good estimate of the convergence time (see Sec. II D). As expected, in both the strong and weak coupling limit the $s^{*}(t)$ converges to $e^{-\lambda^{*} t}$ much earlier than mixing time scales. Therefore, in these regimes, one finds that the convergence time is basically $1 / \lambda^{*}$. On the other hand, for $\sigma=2$ the matching between $e^{-\lambda^{*} t}$ and $s^{*}(t)$ happens only at longer times. Therefore, as expected from the analysis of Sec. II D, in this regime there is a correction to the mixing time due to the norm of the left and right eigenvectors. Nonetheless, similarly to the Liovillian gap, our numerical simulations for small values of $L$ and $q$ show that also the singular value gap is independent of $q$ over the whole range of $\sigma$. Therefore, we argue that it may be a general feature of this model that the resulting convergence time is independent of $q$.

Finally, we consider a stochastic simulation of the evolution of a controllable chain with random fields: we

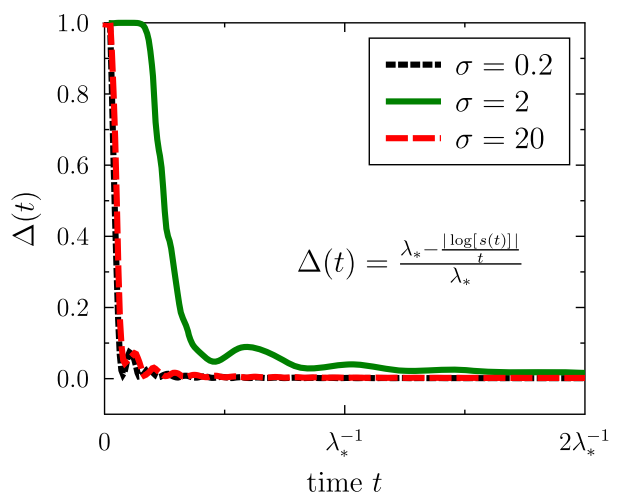

FIG. 5. Convergence of the singular value $s_{*}(t)$ of $e^{t \mathcal{L}} e^{t \mathcal{L}^{\dagger}}$ to $e^{-\lambda_{*} t}$, where $\lambda_{*}$ is the Liouvillian gap. The relative error $\Delta(t)$ between $\lambda_{*}$ and $-t^{-1} \log s(t)$ is plotted for the different values of $\sigma$; the time axis is rescaled between 0 and $2 \lambda_{*}^{-1}$. In the simulations, $L=10, q=1$. generate several random driving functions Eq. (2) and, for each function, we calculate the corresponding unitary evolution and then study the statistics of the generated unitary matrices. To test whether the resulting distribution approximates the Haar measure, we decompose each unitary into the $L^{2}$ angles introduced in Ref. [69]. Using a simple reparametrization of these angles one can write the Haar measure as

$$
d U\left(\varphi_{1}, \ldots, \varphi_{L^{2}}\right)=\prod_{j=1}^{L^{2}} d \varphi_{j},
$$

namely, as a uniform distriution of the angles $\varphi_{j}$ in the range $[0,2 \pi]$. Therefore, testing whether the resulting distribution approximates a Haar measure is equivalent to testing whether the angles $\varphi_{j}$ are distributed as a multinomial uniform distribution. In Fig. 6 we do a simple test to verify the distribution of the angles $\varphi_{j}$ : we divide the interval $[0,2 \pi]$ into 25 bins and plot, as a 3D histrogram, the matrix whose elements $(i, j)$ are the number of times that the angle $\varphi_{i}$ is found in the jth bin. As Fig. 6 shows, the distribution of the unitary matrices is far from uniform both in the noncontrollable case and in the controllable case after a short time (upper panel). Nonetheless, in spite of the finite number of samples, after a long time $(t \approx 55)$ in the
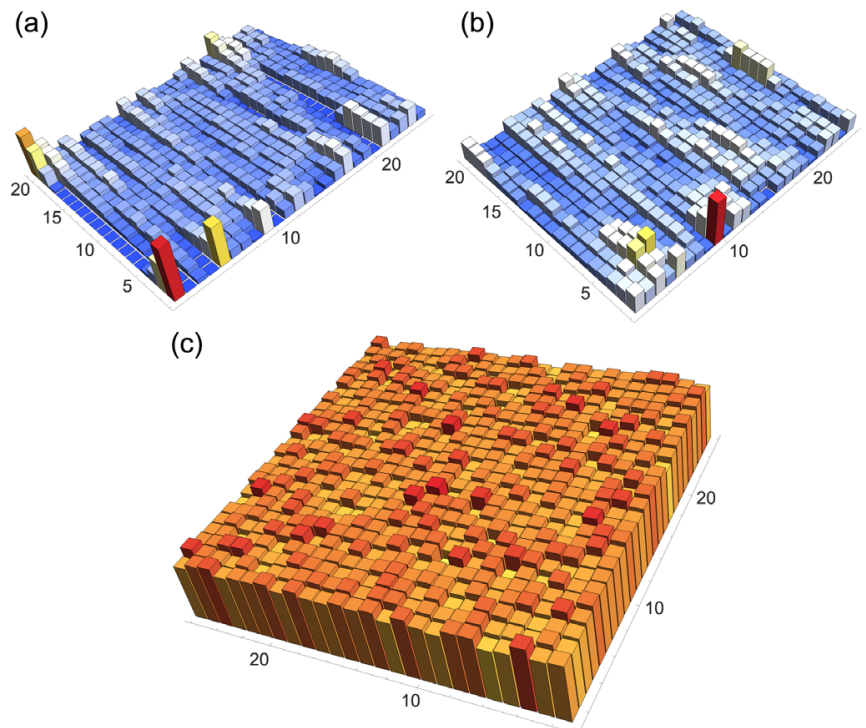

FIG. 6. Uniformity check of generated random unitaries. We consider the time evolution of a driven $L=5$ chain with random fields [Eq. (2)], where $K=100, g_{k}$ is sampled uniformly in $[-0.5,0.5]$, while $\phi_{k}$ and $\omega_{k}$ are sampled in $[-L, L]$. The statistics is done with $10^{4}$ independent realizations. The discrete histrogram is computed according to the decomposition [69] as described in the main text. (a) Noncontrollable case where noise is applied on the central site for a time $t=25$. (b) Controllable case where the noise is applied on the first site for a short time $t=5$. (c) Controllable case where the noise is applied on the first site for a long time $t=55$. 
controllable case the angles' distribution is almost flat (lower panel), thus showing that the resulting unitary matrices are approximately distributed according to the Haar measure.

\section{OTHER APPLICATIONS}

\section{A. Multipoint correlation functions}

Here, we discuss some direct applications, beyond $q$-design, of the main findings of our paper. In boson sampling experiments the output probability is proportional to $|\operatorname{per}(\tilde{U})|^{2}$, with $\operatorname{per}(\tilde{U})$ the matrix permanent of the $q \times q$ matrix $\tilde{U}$, where $\tilde{U}$ is built from some columns and rows of a $L \times L$ Haar-uniform matrix $U$ [24-26]. Therefore,

$$
\begin{aligned}
|\operatorname{per}(\tilde{U})|^{2} & =\sum_{\sigma, \sigma^{\prime}} \prod_{i, j=1}^{q} \tilde{U}_{i, \sigma(i)} \tilde{U}_{j, \sigma^{\prime}(j)}^{*} \\
& =\operatorname{Tr}\left[\mathcal{U}^{\otimes q, q} \mathcal{K}_{\mathrm{BS}}\right],
\end{aligned}
$$

where $\sigma, \sigma^{\prime}$ are permutations in the symmetric group $S_{q}$, $\mathcal{K}_{\mathrm{BS}}$. is a suitable index contraction operator, and $U^{\otimes q, q}=$ $U^{\otimes q} \otimes\left(U^{\otimes q}\right)^{*}$ as in Eq. (4).

A similar expression arises in the evaluation of multipoint correlation functions in quasifree particle-preserving bosonic and fermionic models. If $U$ is the $L \times L$ oneparticle evolution matrix from time 0 to time $t$ and $a_{j}(t)=\sum_{k} U_{j k} a_{k}(0)$, then because of the Wick's theorem,

$$
\left\langle a_{i_{1}}^{\dagger}(t) a_{j_{1}}(t) \ldots a_{i_{q}}^{\dagger}(t) a_{j_{q}}(t)\right\rangle=\operatorname{Tr}\left[U^{\otimes q, q} \mathcal{K}_{\mathrm{MP}}\right],
$$

where $\mathcal{K}_{\mathrm{MP}}$ depends on the initial two-point correlation functions $\left\langle a_{i}^{\dagger}(0) a_{j}(0)\right\rangle$. Expressions like Eq. (53) arise also in $X Y$ spin chains, which can be mapped to a quasifree fermionic model via the Jordan-Wigner transformation [70]. For instance, the driven $X Y$ model,

$$
H_{X Y}(t)=\frac{1}{2}\left[\sum_{j}\left(\sigma_{j}^{x} \sigma_{j+1}^{x}+\sigma_{j}^{y} \sigma_{j+1}^{y}\right)+g(t) \sigma_{1}^{z}\right],
$$

can be mapped, in the single-particle subspace, to the driven quantum walk of Sec. IV. Calling $U$ the resulting single-particle evolution, then in any subspace long-range spin operators $S_{i}^{\alpha} S_{j}^{\beta}$, for $\alpha, \beta \in\{x, y\}$ can be written as a combination of fermion strings as in Eq. (53), where $q=$ $|i-j|$ for $i \neq j$. Therefore, with a suitable $\mathcal{K}_{X Y}$ that depends on the initial correlations, one can write the dynamical long-range correlations between spin operators in an $X Y$ chain as

$$
\left\langle S_{i}^{\alpha}(t) S_{i+q}^{\beta}(t)\right\rangle=\operatorname{Tr}\left[U^{\otimes q, q} \mathcal{K}_{X Y}\right],
$$

for $\alpha, \beta \in\{x, y\}$. Similarly, $\left\langle S_{i}^{z}(t) S_{j}^{z}(t)\right\rangle=\operatorname{Tr}\left[U^{\otimes 2,2} \mathcal{K}_{X Y}^{z z}\right]$.
In all the above cases we can bound the convergence of the random dynamics to the values expected from the Haar distribution. Indeed, for any $\mathcal{K}$,

$$
\begin{aligned}
\mid \operatorname{Tr} & {\left[\left(\mathbb{E}_{U} U^{\otimes q, q}-\int U^{\otimes q, q} \mu_{\text {Haar }}(d U)\right) K\right] \mid } \\
& <e\left(\mu_{U}, q\right)\|K\|_{1},
\end{aligned}
$$

where we use Eq. (4). Thanks to the analysis of Sec. II D, and since the gap Eq. (39) for the controllable quantum walk is independent of $q$, one can then bound the expected errors in all the above cases. For boson sampling experiments, this shows how the error depends on the number $q$ of bosons, while for $X Y$ spin chains it shows how the error decays as a function of the distance $q$ between spins.

\section{B. Estimation of the control time}

We show here that the mixing time, which is easy to compute especially for $q=1$, can give an estimation of the control time. Fixing $H$ and $V$, for how long does one have to drive the system in order to achieve a generic target gate? If after the time $T_{\mathrm{ex}}^{*}$ the random evolutions are Haarrandomly distributed, then the control time to obtain a certain gate $U$ satisfies $T_{c}(U)<T_{\mathrm{ex}}^{*}$. However, for approximate $q$-design, $T^{*}$ provides only a rate of convergence, rather than a sharp bound. This results in an error, which may also be due to the fact that the target gate $U$ is not achievable yet at time $T^{*}$. However, after a time $\tau T^{*}$ this error probability exponentially decreases as a function of $\tau$. We can thus regard $T^{*}$ as an estimation for $T_{c}$. An estimation of the mixing time $T^{*}$ can be easily obtained for any choice of $H$ and $V$ via the inverse of the gap $\lambda^{*}$, which depends on $\sigma$ (see, e.g., Fig. 3). Since $T_{c}$ does not involve any specific properties (amplitudes, frequencies) of the pulse, one has to compare it with $T_{\min }^{*}=$ $\min _{\sigma} T^{*}(\sigma) \simeq T^{*}(\sigma \simeq 2.5) \approx 0.055 L^{3}$.

In order to estimate $T_{c}$ we perform a numerical experiment with the QuTip quantum control package [71]. We consider the model Eq. (31) and, for each length $L=10, \ldots, 20$, we generate a Haar-random unitary $U$ and find the time $T_{c}$ as the minimal time for which the program converges. We find that $T_{c}$ obtained in this way scales as $T_{c} \approx 0.069 L^{3}$. This shows two remarkable facts: (i) the values of $T_{c}$ and $T_{\min }^{*}$ are very close for $L=10, \ldots, 20$, and (ii) both $T_{c}$ and $T_{\min }^{*}$ exhibit the same scaling with the length $L$, so it is expected that this close relationship is maintained also for larger $L$. In view of our findings, one can find an empirical upper bound on $T_{c}$ as $3 T_{\min }^{*} / 2$.

\section{CONCLUSIONS AND PERSPECTIVES}

In this paper we study the quantum dynamics resulting from a stochastic driving of quantum many-body systems, and we answer the following questions: when, and how 
rapidly, the dynamics of a driven quantum system is equivalent to a fully uniform random evolution, namely under unitaries sampled from the Haar measure. The first major finding is that, when the system is fully controllable and the stochastic signal has finite correlation time, then its random dynamics converges to the Haar distribution in the "long-time" limit. The second major result is about the estimation of the driving time $T^{*}$ : this is done by studying the deviations from the Haar distribution using the framework of approximate $q$-design, and using second quantization to map the problem into the estimation of the mixing time in an open quantum many-body Liouvillian with $2 q$ virtual particles.

We perform a thorough analysis of the Markovian limit (e.g., white noise) using tools from the theory of dynamical semigroups, and we find upper bounds on $T^{*}$ in terms of the gap of the Liouvillian operator. We study the mean-field solution of the resulting many-body model, which predicts a constant Liouvillian gap as a function of $q$, and we show its limitations via symmetry-breaking arguments. Nonetheless, we find that the mean-field predictions are correct in a wide variety of different numerical studies, obtained with random choices of $H$ and $V$, and match with the analytic solution of a particular model, namely, a onedimensional system with strong control on one of its boundaries. The latter analytic solution is obtained by mapping the effective Liovillian to an exactly solvable model, and then using Bethe-ansatz techniques to explicitly show that the excited states with the smallest gap are built from unpaired quasiparticles, as in the mean-field treatment. We then corroborate our predictions with numerical simulations, giving strong evidence that the considered one-dimensional model provides a quantum expander with a constant mixing time as a function of $q$. Therefore, our results show that certain driven physical systems can provide a significant advantages over random quantum circuits where the mixing time increases polynomially as a function of $q$ [35].

The results we present in this paper have many applications. The first one, already discussed, is a physically motivated approach to generate pseudouniform random unitary operations, which have many applications in quantum information processing protocols. The onedimensional system that is extensively analyzed in this paper is motivated by the recent experiments with integrated photonic circuits [21,22], where random unitary operations have been used in the first small-scale experimental observations of boson sampling [24-26]. The results we present in this paper enable the implementation of random operations in integrated photonic chips that, being based on noisy quantum walks rather than carefully designed multimode beam splitters and phase shifters, are much simpler to fabricate for a larger number of modes. Therefore, our results provide a new avenue to prove quantum supremacy in boson sampling experiments.
Moreover, we consider other applications, such as the dynamics of correlation functions in an $X Y$ spin chain, and the estimation of the control time $T_{c}$, one of the major open problems for quantum control. Given a target unitary $U$ and the physical interactions described by $H_{0}$ and $V$, how can we choose $T_{c}$ such that $U$ is achievable by driving the system for a time $T_{c}$ ? With numerical experiments, performed on $L$-site chains, we find that both $T_{c}$ and $T_{*}$ are very close for $L=10, \ldots, 20$, and both scale as $L^{3}$. Hence, the mixing time $T_{*}$ under random signals provides an easily computable estimation of $T_{c}$, for any $H_{0}$ and $V$.

Finally, there are several applications in quantum manybody physics, where the interplay between quantum manybody effects and noise is currently a subject of intensive study in many areas, such as spin glass [42], the fast scrambling of quantum information [28,29], and manybody localization [72,73]. The explicit one-dimensional model discussed in Sec. IV is a single-particle model, where many-body physics arises due to unitary $q$-design, which introduces $2 q$ virtual particles. An interesting future perspective is the study of random driving in physical interacting many-body systems (e.g., interacting spin systems and/or cold atom optical lattices). In fact, the competition between physical many-body effects and those arising from the unitary design, may give rise to novel states of matters and phase transitions [68,74-77], produce a large amount of entanglement [78], and give new insights into the process of thermalization and equilibration [79]. Haar-random quantum states are known to have, typically, an extensive amount of entanglement [80]. Since we show that any controllable quantum system converges to a maximally mixing dynamics, the real-time dynamics will be very hard to simulate numerically in the many-body settings, because of the large amount of entanglement involved. Nonetheless, the controllability requirement provides a sufficient algebraic method to infer $a$ priori whether a randomly driven condensed-matter system is expected to produce a lot of entanglement in the long-time limit.

\section{ACKNOWLEDGMENTS}

The authors thank S. Bose, E. Compagno, F. Falceto, J. Links, A. Marcus, L. Maccone, S. Maniscalco, E. Mukhin, S. Paesani, R. Santagati, S. Severini, A. Werner, R. Zeier, and Z. Zimborás for interesting discussions. L. B. has received funding for this research from the European Research Council under the European Union's Seventh Framework Programme (FP7/2007-2013)/ERC Grant Agreement No. 308253 PACOMANEDIA. M. J. K. was supported by the Villum foundation. D. B. acknowledges support from the EPSRC Grant No. EP/M01634X/1.

\section{APPENDIX A: GAUSSIAN HARMONIC PULSES}

To simplify the theoretical description, in this section we consider only $q=1$ and call $\mathcal{E}_{t}$ the quantum channel 
resulting from the average evolution of the quantum system:

$$
\mathcal{E}_{t}[\rho]=\mathbb{E}\left[\mathcal{T} e^{-i \int_{0}^{t} \mathcal{H}(s) d s} \rho \mathcal{T} e^{i \int_{0}^{t} \mathcal{H}(s) d s}\right] .
$$

Extension to higher values of $q$ is straightforward. As described in Sec. II, we now make two assumptions, namely that $g(t)$ is Gaussian and harmonic, where $\mathbb{E}[g(t+$ $s) g(t)]=c(s)$ is independent of $t$ and $\mathbb{E}[g(t)]=0$. In view of these assumptions, we can simplify Eq. (A1) by expanding the exponentials into the Dyson series, then using the Wick's theorem to decompose the expectation values, and finally resumming the series. The result in the interaction picture is then $[38,39]$

$$
\begin{gathered}
\mathcal{E}_{t}^{(I)}\left[\rho^{(I)}\right]=\mathcal{T} e^{-\int_{0}^{t} d s \mathcal{W}_{s}} \rho^{(I)}, \\
\mathcal{W}_{s} \rho=\int_{0}^{s} c\left(s-s^{\prime}\right)\left[V^{(I)}(s),\left[V^{(I)}\left(s^{\prime}\right), \rho\right]\right] d s^{\prime},
\end{gathered}
$$

where $(I)$ refers to the interaction picture with respect to $H$. If the correlation time is finite, then there exists a suitably large $T$ such that $T c(T s) \simeq(\sigma / 2) \delta(s)$, where $\delta$ is the Dirac delta function and $\sigma$ is a constant. In the long-time limit one finds that

$$
\mathcal{E}_{t}^{(I)}\left[\rho^{(I)}\right] \simeq \mathcal{T} \exp \left(-c \int_{0}^{t}\left[V^{(I)}(s),\left[V^{(I)}(s), \cdot\right]\right] d s\right) \rho^{(I)},
$$

when $t>T$, namely in the Schrödinger picture:

$$
\begin{aligned}
\mathcal{E}_{t}[\rho] & \simeq e^{-t \mathcal{L}} \rho, \\
\mathcal{L} \rho & =-i[H, \rho]-\frac{\sigma}{2}[V,[V, \rho]] .
\end{aligned}
$$

\section{APPENDIX B: SEMIGROUP CONVERGENCE TIMES}

There exist several measures to estimate convergence of a semigroup of completely positive trace-preserving maps. The one with the most natural operational interpretation is trace norm convergence, as it reflects the likelihood that the time-evolved state can be distinguished from the stationary state at a given time $t$.

$$
\sup _{\rho}\left\|e^{t \mathcal{L}}(\rho)-T_{\infty}(\rho)\right\|_{1} \leq \epsilon(t)
$$

where $T_{\infty}=\lim _{t \rightarrow \infty} e^{t \mathcal{L}}$, and $\epsilon(t)$ is the distinguishability error. A less stringent convergence requirement is to ask whether $e^{t \mathcal{L}^{q}}$ is an expander for a given value of $t$. Then, we want to estimate

$$
\left\|e^{t \mathcal{L}}-T_{\infty}\right\|_{2 \rightarrow 2}=\left\|e^{t \hat{\mathcal{L}}^{q}}-\hat{T}_{\infty}\right\|_{\infty},
$$

where a hat indicates that the completely positive tracepreserving maps are represented as channels (see Ref. [81] for more details on the representation of channels). Trace norm convergence and "spectral convergence" are related, by noting that

$$
\left\|e^{t \mathcal{L}}-T_{\infty}\right\|_{2 \rightarrow 2} \leq\left\|e^{t \mathcal{L}}-T_{\infty}\right\|_{1 \rightarrow 1} \leq d^{2}\left\|e^{t \mathcal{L}}-T_{\infty}\right\|_{2 \rightarrow 2},
$$

where $d$ is the dimension of the Hilbert space, and recalling that $\left\|e^{t \mathcal{L}}-T_{\infty}\right\|_{1 \rightarrow 1}=\sup _{\rho}\left\|e^{t \mathcal{L}}(\rho)-T_{\infty}(\rho)\right\|_{1}$.

In order to estimate the above norms it is important to recall the spectral properties of quantum dynamical semigroups. The spectrum of a Liouvillian $\mathcal{L}$ has a nonpositive real part, and there always exists at least one eigenvalue of magnitude zero, corresponding to a stationary state of the semigroup: $\mathcal{L}(\rho)=0$. The rest of the spectrum comes in complex conjugate pairs. The Liouvillian is called unital if it annihilates the identity $\mathcal{L}(\mathbb{1})=0$. The Liouvillian in Eq. (7) has this property. A unital Liouvillian is called reversible if $\hat{\mathcal{L}}=\hat{\mathcal{L}}^{\dagger}$, in which case its spectrum is real. Unfortunately, Eq. (7) is not reversible. Convergence of a nonreversible semigroup is governed by the singular values of $e^{t \mathcal{L}}$ rather than its eigenvalues. The singular spectrum of $e^{t \mathcal{L}}$ is equal to the spectrum of $\sqrt{e^{t \hat{\mathcal{L}}} e^{t \hat{\mathcal{L}}^{\dagger}}}$.

It is not difficult to see that the $2 \rightarrow 2$ norm is related to the singular spectrum. Let $s_{j}(t)$ be the singular values of $e^{t \mathcal{L}}$, ordered from largest to smallest. The largest has magnitude one. We know that asymptotically $s^{*}(t)=e^{t \operatorname{Re}\left[\lambda^{*}\right]}$, where now $\lambda_{j}$ are the eigenvalues of $\mathcal{L}$ written in decreasing (real part) order, and $\lambda^{*}$ is the gap of $\mathcal{L}$, i.e., the smallest (in magnitude) nonzero real part of any eigenvalue of $\mathcal{L}$. To see this, note that, assuming it has no Jordan blocks, the Liouvillian can be written in its spectral decomposition as

$$
\mathcal{L}(\rho)=\sum_{j} \lambda_{j} L_{j}^{\dagger} \operatorname{tr}\left[R_{j} \rho\right],
$$

where $R_{j}, L_{j}$ are a biorthonormal basis of operators: i.e., $\operatorname{tr}\left[L_{j}^{\dagger} R_{k}\right]=\delta_{j k}$. Importantly, the norm of any given $L_{j}, R_{j}$ can be large, which prevents us from getting any rigorous (universal) bounds between the singular values and the eigenvalues. Then,

$$
\begin{aligned}
& \left\|e^{t \mathcal{L}}-T_{\infty}\right\|_{2 \rightarrow 2} \\
& =\sup _{\psi}\left(\sum_{j: \lambda_{j} \neq 0} e^{2 t \operatorname{Re}\left[\lambda_{j}\right]}\left|\left\langle R_{j} \mid R_{j}\right\rangle\right|\left|\left\langle\psi \mid L_{j}\right\rangle\left\langle L_{j} \mid \psi\right\rangle\right|\right)^{1 / 2} \\
& \approx_{t \rightarrow \infty} e^{t \lambda^{*}}\left(\left|\left\langle R_{j} \mid R_{j}\right\rangle \|\left\langle\psi \mid L_{j}\right\rangle\left\langle L_{j} \mid \psi\right\rangle\right|\right)^{1 / 2} .
\end{aligned}
$$

Hence, for very large $t$, the convergence is governed by the gap, and $s^{*}(t) \rightarrow e^{t \lambda^{*}}$. In principle we do not know at what scale $e^{-t \lambda^{*}} \gg\left|\left\langle R_{j} \mid R_{j}\right\rangle\right|\left|\left\langle\psi \mid L_{j}\right\rangle\left\langle L_{j} \mid \psi\right\rangle\right|$. 
We argue in the main text that for the specific model of a controllable quantum walk, the prefactors do not contribute to the asymptotics in the weak- or strong-coupling limits.

\section{APPENDIX C: WEAK-DRIVING LIMIT}

A convenient approximation for the long-time dynamics in the weak-coupling limit $\sigma \ll 1$ is the rotating wave approximation (RWA) [82]. We consider the case $q=1$ and assume that $V$ is a matrix of real numbers and call $\mathcal{D}=-\sigma \stackrel{\circ}{V}^{2} / 2$ the dissipative part in Eq. (8). Going to the interaction picture with respect to the Hamiltonian part, one finds that $\dot{\rho}_{I}(t)=\mathcal{D}_{I}(t) \rho_{I}(t)$, where in the eigenbasis of $H=\sum_{j} \omega_{j}\left|\omega_{j}\right\rangle\left\langle\omega_{j}\right|$ it is

$$
\begin{gathered}
\left\langle\omega_{i} \omega_{j}\left|\mathcal{D}_{I}(t)\right| \omega_{k} \omega_{l}\right\rangle=-\frac{\sigma}{2} e^{-i t\left(\omega_{i j}-\omega_{k l}\right)} \mathcal{R}_{i j k l}, \\
R_{i j k l}=\left\langle\omega_{i}\left|V^{2}\right| \omega_{k}\right\rangle \delta_{j l}+\left\langle\omega_{j}\left|V^{2}\right| \omega_{l}\right\rangle \delta_{i k} \\
-2\left\langle\omega_{i}|V| \omega_{k}\right\rangle\left\langle\omega_{j}|V| \omega_{l}\right\rangle,
\end{gathered}
$$

where $\omega_{i j}=\omega_{i}-\omega_{j}$. The rotating wave approximation consists in neglecting all the terms where $\omega_{i j} \neq \omega_{k l}$, because for large $t$ they are highly oscillating and average out:

$$
\left\langle\omega_{i} \omega_{j}\left|\mathcal{D}^{\mathrm{RWA}}\right| \omega_{k} \omega_{l}\right\rangle=R_{i j k l} \delta_{\omega_{i j}, \omega_{k l}}
$$

This approximation is expected to hold when

$$
t \gg \max _{\omega_{i j} \neq \omega_{k l}}\left(\omega_{i j}-\omega_{k l}\right)^{-1} .
$$

RWA is related to degenerate perturbation theory. Indeed, the unperturbed ( $\sigma=0)$ eigenvalues of Eq. (8) are given by $\left|\Phi_{i j}^{(0)}\right\rangle=\sum_{k l} \delta_{\omega_{i j}, \omega_{k l}} \alpha_{k l}^{i j}\left|\omega_{k} \omega_{l}\right\rangle$ with eigenvalue $-i \omega_{i j}$. From degenerate first-order perturbation theory, we know that, for small $\sigma$, the eigenvalues of Eq. (8) are obtained by diagonalizing $\mathcal{D}^{\mathrm{RWA}}$, which is block diagonal where each block acts on different degenerate subspaces. The eigenvectors of $\mathcal{D}^{\mathrm{RWA}}$ provide the matrices $\alpha_{k l}^{i j}$. Note that since $\mathcal{D}^{\mathrm{RWA}}$ is Hermitian, the states $\left|\Phi_{i j}^{(0)}\right\rangle$ form an orthonormal basis that depends on both $H$ (from the basis $\left|\omega_{k}\right\rangle$ ) and $V$ (via the diagonalization of $\mathcal{D}^{\mathrm{RWA}}$ ). Moreover, the real eigenvalues $\Delta_{i j}$ of Eq. (C3) provide the first-order correction to the eigevectors of Eq. (8) that, to the first order in $\sigma^{-1}$, are $-i \omega_{i j}+\Delta_{i j}$. The Liouvillian gap is given by the minimum nonzero value of $-\Delta_{i j}$. Similarly, one finds the correction to the (right) eigenvector:

$$
\begin{aligned}
\left|\Phi_{i j}^{(1)}\right\rangle & =\left|\Phi_{i j}^{(0)}\right\rangle-i \sum_{\substack{k l \\
\omega_{k l} \neq \omega_{i j}}}\left|\Phi_{k l}^{(0)}\right\rangle \frac{\left\langle\Phi_{k l}^{(0)}|\mathcal{D}| \Phi_{i j}^{(0)}\right\rangle}{\omega_{k l}-\omega_{i j}} \\
& \simeq e^{\mathcal{S}^{\mathrm{RWA}}}\left|\Phi_{i j}^{(0)}\right\rangle,
\end{aligned}
$$

where

$$
\mathcal{S}^{\mathrm{RWA}}=-i \sum_{\substack{k l m n \\ \omega_{k l} \neq \omega_{i j}}}\left|\Phi_{k l}^{(0)}\right\rangle \frac{\left\langle\Phi_{k l}^{(0)}|\mathcal{D}| \Phi_{m n}^{(0)}\right\rangle}{\omega_{k l}-\omega_{m n}}\left\langle\Phi_{m n}^{(0)}\right| .
$$

Since $\mathcal{S}^{\mathrm{RWA}}$ is a Hermitian operator, the new vectors in Eq. (C5) do not form an orthonormal basis.

We now focus on the chain discussed in Sec. IV, where $\omega_{k_{j}}=2 \cos k_{j}, k_{j}=\pi j /(L+1), V^{2}=V$, and we call $W_{i j}=\left\langle\omega_{i}|V| \omega_{j}\right\rangle=(2 / L+1) \sin k_{i} \sin k_{j}$. To simplify the equations we use the compact notation $|i\rangle \equiv\left|\omega_{i}\right\rangle$ and we use $c=1$, namely, we assume that the controlled site is the first one. We note that the resonance condition $\omega_{i}-\omega_{j}=\omega_{k}-\omega_{l}$ is achieved in three different cases:

Case 1: $i=k$ and $j=l$ :

$$
\left\langle i j\left|\mathcal{D}^{\mathrm{RWA}}\right| i j\right\rangle=\frac{\sigma}{2}\left(V_{i i}+V_{j j}-2 V_{i i} V_{j j}\right) .
$$

Case 2: $i=j \neq k=l$ :

$$
\left\langle i i\left|\mathcal{D}^{\mathrm{RWA}}\right| k k\right\rangle=\frac{\sigma}{2}\left(-2 V_{i k}^{2}\right) .
$$

Case 3: We note that $\omega_{i}+\omega_{\bar{i}}=0$, where $\bar{i}=L-i+1$. Therefore, if $l=\bar{i}$ and $k=\bar{j}$, the resonance condition is achieved. To avoid double counting with case 1 , we write $l=\bar{i}, k=\bar{j}, i \neq j, i \neq \bar{j}$, so

$$
\left\langle i j\left|\mathcal{D}^{\mathrm{RWA}}\right| \bar{j} \bar{i}\right\rangle=\frac{\sigma}{2}\left(-2 V_{i \bar{j}} V_{j \bar{i}}\right)=\frac{\sigma}{2}\left(-2 V_{i j}^{2}\right),
$$

where we use the fact that $V_{i j}=V_{j i}=V_{\bar{i} j}=V_{j i}$. All the other elements are zero.

All the nonzero elements of $\mathcal{D}^{\mathrm{RWA}}$ are discussed in cases $1-3$. Since most of the terms are zero, it is quite easy to find the eigenvalues of $\mathcal{D}^{\mathrm{RWA}}$. We call those eigenvalues $|S\rangle=\sum_{i j} S_{i j}|i j\rangle$. From cases 1 and 3 , one can see that the off-diagonal states where $S_{i i}=0$ are decoupled from the diagonal ones. Therefore, we consider these two cases separately. Let $\left|S_{o}\right\rangle=\sum_{i \neq j} S_{i j}|i j\rangle$ be an off-diagonal state, then the eigenvalue equation $\mathcal{D}^{\mathrm{RWA}}\left|S^{o}\right\rangle=\lambda\left|S^{\circ}\right\rangle$ written as $\left\langle k l\left|\mathcal{D}^{\mathrm{RWA}}\right| S^{o}\right\rangle=\lambda S_{k l}$ for $k \neq l$ is

$$
\left(V_{k k}+V_{l l}-2 V_{k k} V_{l l}\right) S_{k l}-2 V_{k l}^{2} S_{\bar{l} \bar{k}}=-\frac{2}{\sigma} \lambda_{k l} S_{k l},
$$

when $l \neq \bar{k}$ and 


$$
\left(2 V_{k k}-2 V_{k k}^{2}\right) S_{k \bar{k}}=-\frac{2}{\sigma} \lambda_{k \bar{k}} S_{k \bar{k}}
$$

Therefore, for each pair $k, l$, Eq. (C10) is a $2 \times 2$ matrix eigenvalue problem whose minimum (in absolute value) eigenvalue is

$$
\begin{aligned}
\lambda_{k l}^{\min } & =-\frac{\sigma}{2}\left(V_{k k}+V_{l l}-4 V_{k k} V_{k l}\right) \\
& =-\frac{\sigma}{L+1}\left(\sin ^{2} k_{k}+\sin ^{2} k_{l}-\frac{8}{L+1} \sin ^{2} k_{k} \sin ^{2} k_{l}\right) .
\end{aligned}
$$

On the other hand,

$$
\begin{aligned}
\lambda_{\bar{l} \bar{l}} & =-\frac{\sigma}{2}\left(2 V_{l l}-2 V_{l l}^{2}\right) \\
& =-\frac{2 \sigma}{L+1}\left(\sin ^{2} k_{l}-\frac{2}{L+1} \sin ^{4} k_{l}\right) .
\end{aligned}
$$

When $L \gg 1$ we can neglect the $\mathcal{O}\left(L^{-2}\right)$ correction, and since $V_{l l}$ is minimized for $l=1$, we find that the gap is

$$
\operatorname{gap}=-\lambda_{1 \overline{1}} \approx \frac{2 \sigma \pi}{L^{3}}
$$

We now show that the other "diagonal" eigenvalues $\left|S^{d}\right\rangle=\sum_{i} S_{i i}|i i\rangle$ have a larger gap. Writing the eigenvalue equation, we find $-(\sigma / 2)\left(2 V_{k i} \delta_{k i} S_{k k}-2 V_{k i}^{2} S_{i i}\right)=\lambda S_{k k}$; namely, we have to find the eigenvalues of the matrix $R_{i k}=\sigma\left(V_{i k} \delta_{i k}-V_{i k}^{2}\right)$. Calling $V^{d}=\sigma \operatorname{diag} V$ and $a_{i}=$ $(2 \sqrt{\sigma} / L+1) \sin ^{2} k_{i}$, then $R=-V^{d}+a^{T} a$. Using the matrix determinant lemma in the eigenvalue equation, we find

$$
\begin{aligned}
0 & =\operatorname{det}\left(\lambda \mathbb{1}+V^{d}-a^{T} a\right) \\
& =\operatorname{det}\left(\lambda \mathbb{1}+V^{d}\right)\left(1-a^{T} \frac{1}{\lambda \mathbb{1}+V^{d}} a\right) .
\end{aligned}
$$

The first term in the above equation gives the solutions $\lambda=-V_{l l}=-(2 \sigma / L+1) \sin ^{2} k_{l}$, which have a higher gap. On the other hand, the second term in Eq. (C13) provides the equation

$$
\begin{aligned}
0 & =1-\frac{4 \sigma}{(L+1)^{2}} \sum_{l} \frac{\sin ^{4} k_{l}}{\lambda+\frac{2 \sigma}{L+1} \sin ^{2} k_{l}} \\
& =1-\frac{2}{(L+1)} \sum_{l} \frac{\sin ^{4} k_{l}}{\frac{L+1}{2 \sigma} \lambda+\sin ^{2} k_{l}} \\
& =1-\frac{2}{(L+1)} \sum_{l}\left(\sin ^{2} k_{l}+\frac{L+1}{2 \sigma} \lambda \frac{\sin ^{2} k_{l}}{\frac{L+1}{2 \sigma} \lambda+\sin ^{2} k_{l}}\right)
\end{aligned}
$$

where in the last equation we use the identity $(1 / a+b)=(1 / a)-[b / a(a+b)]$. Since $\sum_{l} \sin ^{2} k_{l}=$ $(L+1) / 2$, we are left with the equation

$$
0=\lambda \sum_{l} \frac{\sin ^{2} k_{l}}{\frac{L+1}{2 \sigma} \lambda+\sin ^{2} k_{l}}
$$

A solution to that equation is clearly $\lambda=0$, namely, the steady state. On the other hand, all the other solutions must satisfy $\lambda<-(2 \sigma / L+1) \sin ^{2} k_{l}$ for some $l$, because otherwise all the elements in the sum are positive and there is clearly no solution. Therefore, all the solutions must satisfy $|\lambda|>(2 \sigma / L+1) \sin ^{2} k_{1}>$ gap. This concludes the proof that the gap is given by Eq. (C12).

\section{APPENDIX D: STRONG-DRIVING LIMIT}

We focus here on the derivation of the effective Liouvillian Eq. (33). Let us then define $\mathcal{P}$ as the projector onto the low-energy (eigenvalue zero) subspace of $\mathcal{D}=\frac{1}{2}\left(n_{1}^{\uparrow}-n_{1}^{\downarrow}\right)\left(n_{1}^{\uparrow}-n_{1}^{\downarrow}\right)$. This space is generated by all the states such that $n_{1}^{\uparrow}=n_{1}^{\downarrow}$. We also set $\mathcal{Q}=\mathbb{1}-\mathcal{P}$ and call $\mathcal{H}$ the Hamiltonian part such that $\mathcal{L}_{q}=-i \mathcal{H}-\sigma \mathcal{D}$. We then also call $X_{P P}=\mathcal{P} X \mathcal{P}$, with similar definitions for $X_{P Q}, X_{Q P}, X_{Q Q}$. We can therefore write $\mathcal{L}_{q}$ in the block form:

$$
\mathcal{L}_{q}=\left(\begin{array}{cc}
-i \mathcal{H}_{P P} & -i \mathcal{H}_{P Q} \\
-i \mathcal{H}_{Q P} & -i \mathcal{H}_{Q Q}-\sigma \mathcal{D}_{Q Q}
\end{array}\right)
$$

where $\sigma \gg\|\mathcal{H}\|,\|\mathcal{D}\|$, and where we use the fact that $\mathcal{P D}=\mathcal{D} \mathcal{P}=0$. The low-energy eigenvalues can then be obtained using the determinant identity

$$
\operatorname{det}\left(\begin{array}{ll}
A & B \\
C & D
\end{array}\right)=\operatorname{det}(D) \operatorname{det}\left(A-B D^{-1} C\right)
$$

see also Refs. [46,83] for a related approach. Indeed, using a first-order expansion for $\sigma \rightarrow \infty$, it is simple to see that the small eigenvalues are the eigenvalues of the effective operator:

$$
\mathcal{L}_{q}^{\mathrm{eff}}=-i \mathcal{H}_{P P}-\frac{1}{\sigma} \mathcal{H}_{P Q} \mathcal{D}_{Q Q}^{-1} \mathcal{H}_{Q P}
$$

The above effective operator can also be obtained with a (possibly nonunitary) similarity transformation $e^{\mathcal{S}_{D}}$ to decouple the "low-energy" and "high-energy" subspaces. Namely, one can find $\mathcal{S}_{D}$ such that

$$
\begin{aligned}
\left(\begin{array}{cc}
\mathcal{L}_{q}^{\text {eff }} & 0 \\
0 & \mathcal{O}(\sigma)
\end{array}\right)= & e^{\mathcal{S}_{D}} \mathcal{L}_{q} e^{-\mathcal{S}_{D}} \\
= & \mathcal{L}_{q}+\left[\mathcal{S}_{D}, \mathcal{L}_{q}\right]+\frac{\left[\mathcal{S}_{D},\left[\mathcal{S}_{D}, \mathcal{L}_{q}\right]\right]}{2} \\
& +\mathcal{O}\left(\left\|\mathcal{S}_{D}\right\|^{3}\right) .
\end{aligned}
$$

One finds that Eq. (D3) is valid up to the first order in $\sigma^{-1}$, 
with $\mathcal{L}_{q}^{\text {eff }}$ given by Eq. (D2), by choosing

$$
\mathcal{S}_{D}=\frac{\mathcal{S}_{1}}{\sigma}+\frac{\mathcal{S}_{2}}{\sigma^{2}}+\mathcal{O}\left(\sigma^{-3}\right)
$$

such that

$$
\begin{aligned}
\mathcal{S}_{1} & =\left(\begin{array}{cc}
0 & i \mathcal{H}_{P Q} \mathcal{D}_{Q Q}^{-1} \\
-i \mathcal{D}_{Q Q}^{-1} \mathcal{H}_{Q P} & 0
\end{array}\right), \\
\mathcal{S}_{2} & =\left(\begin{array}{cc}
0 & \mathcal{S}_{2, *} \\
-\mathcal{S}_{2, *}^{\dagger} & 0
\end{array}\right),
\end{aligned}
$$

where $\mathcal{S}_{2, *}=\mathcal{H}_{P P} \mathcal{H}_{P Q} \mathcal{D}_{Q Q}^{-2}-\mathcal{H}_{P Q} \mathcal{D}_{Q Q}^{-1} \mathcal{H}_{Q Q} \mathcal{D}_{Q Q}^{-1}$. Note that $i \mathcal{S}_{1}$ is a Hermitian operator, unlike $i \mathcal{S}_{2}$.

We now obtain the effective operator explicitly. Since $\mathcal{P}$ commutes with all the operators acting on all but the first sites, one realizes that $\mathcal{H}_{P Q}$ and $\mathcal{H}_{P Q}$ are composed only by the projections of $a_{1 \uparrow}^{\dagger} a_{2 \uparrow}$ and their complex conjugate. Moreover,

$$
\begin{aligned}
\mathcal{P} a_{1 \uparrow}^{\dagger} \mathcal{Q}= & \sum_{n_{1 \uparrow}} \sum_{m_{1 \uparrow} \neq m_{1 \downarrow}}\left|n_{1 \uparrow} n_{1 \uparrow}\right\rangle\left\langle n_{1 \uparrow} n_{1 \uparrow}\left|a_{1 \uparrow}^{\dagger}\right| m_{1 \uparrow} m_{1 \downarrow}\right\rangle \\
& \times\left\langle m_{1 \uparrow} m_{1 \downarrow}\right| \\
= & \sum_{n_{1}} \sqrt{n_{1}}\left|n_{1}, n_{1}\right\rangle\left\langle n_{1}-1, n_{1}\right|
\end{aligned}
$$

where $|m n\rangle$ is a shorthand notation for $\left[\left(a_{1 \uparrow}^{\dagger}\right)^{m}\left(a_{1 \downarrow}^{\dagger}\right)^{n}\right] /$ $[\sqrt{m ! n !}]|0\rangle$. Similarly, we find

$$
\begin{array}{r}
\mathcal{P} a_{1 \downarrow}^{\dagger} \mathcal{Q}=\sum_{n_{1}} \sqrt{n_{1}}\left|n_{1}, n_{1}\right\rangle\left\langle n_{1}, n_{1}-1\right|, \\
\mathcal{P} a_{1 \uparrow} \mathcal{Q}=\sum_{n_{1}} \sqrt{n_{1}}\left|n_{1}, n_{1}\right\rangle\left\langle n_{1}+1, n_{1}\right|, \\
\mathcal{P} a_{1 \downarrow} \mathcal{Q}=\sum_{n_{1}} \sqrt{n_{1}+1}\left|n_{1}, n_{1}\right\rangle\left\langle n_{1}, n_{1}+1\right| .
\end{array}
$$

Since in $\mathcal{H}_{Q P}$ the up and down states on the first site differ for only one paritcle, it is $\mathcal{D}_{Q Q}^{-1} \mathcal{H}_{Q P}=2 \mathcal{H}_{Q P}$. Hence, the effective operator is given by $-i \mathcal{H}_{P P}-(2 / \sigma) \mathcal{H}_{P Q} \mathcal{H}_{Q P}$. This can be computed from

$$
\begin{gathered}
\mathcal{P} a_{1 \uparrow}^{\dagger} \mathcal{Q} a_{1 \uparrow} \mathcal{P}=n_{1 \uparrow} \mathcal{P}, \\
\mathcal{P} a_{1 \uparrow} \mathcal{Q} a_{1 \uparrow}^{\dagger} \mathcal{P}=\left(n_{1 \uparrow}+1\right) \mathcal{P}, \\
\mathcal{P} a_{1 \uparrow}^{\dagger} \mathcal{Q} a_{1 \downarrow}^{\dagger} \mathcal{P}=a_{1 \uparrow}^{\dagger} a_{1 \downarrow}^{\dagger} \mathcal{P},
\end{gathered}
$$

and their Hermitian conjugate (all the other terms are zero). Moreover, $n_{1 \uparrow} \mathcal{P}=n_{1 \downarrow} \mathcal{P}$. We find then

$$
\begin{aligned}
\mathcal{H}_{P Q} \mathcal{H}_{Q P}= & -2\left(a_{1 \uparrow}^{\dagger} a_{1 \downarrow}^{\dagger} a_{2 \uparrow} a_{2 \downarrow}+\text { H.c. }\right)+n_{1 \uparrow}\left(n_{2 \downarrow}+1\right) \\
& +n_{1 \downarrow}\left(n_{2 \uparrow}+1\right)+n_{2 \downarrow}\left(n_{1 \uparrow}+1\right)+n_{2 \uparrow}\left(n_{1 \downarrow}+1\right)
\end{aligned}
$$

$$
\begin{aligned}
= & -2\left(a_{1 \uparrow}^{\dagger} a_{1 \downarrow}^{\dagger} a_{2 \uparrow} a_{2 \downarrow}+\text { H.c. }\right)-1 \\
& +\left(n_{1 \uparrow}+n_{1 \downarrow}+1\right)\left(n_{2 \uparrow}+n_{2 \downarrow}+1\right) .
\end{aligned}
$$

In order to make further analytical progress we also use the rotating wave approximation, which is consistent with the perturbative treatment (see Appendix C) since $\mathcal{L}_{q}^{\text {eff }}=$ $-i \mathcal{H}_{P P}-(2 / \sigma) \mathcal{H}_{P Q} \mathcal{H}_{Q P}$ and $2 / \sigma$ is small. We note that $\mathcal{H}_{P P}=\sum_{\alpha=2}^{L}\left(a_{\alpha \uparrow}^{\dagger} a_{\alpha+1, \uparrow}-a_{\alpha \downarrow}^{\dagger} a_{\alpha+1, \downarrow}+\right.$ H.c. $)$. The above operator can be diagonalized with a Bogoliubov transformation: defining the operators $\tilde{a}_{k \uparrow}=$ $\sum_{\alpha=1}^{L-1}(2 / L) \sin ^{2}(\pi k \alpha / L) a_{\alpha+1, \uparrow}$, we find that $\mathcal{H}_{P P}=$ $\sum_{k=1}^{L} 2 \cos (k \pi / L)\left(\tilde{n}_{k \uparrow}-\tilde{n}_{k \downarrow}\right)$. Because of this particular form, the rotating wave approximation in Eq. (D12) corresponds to expanding the operators $a_{2 \uparrow}$ into the diagonal basis $\tilde{a}_{k \uparrow}$, neglecting the "oscillating" off-diagonal terms. In other terms, we can write

$$
\mathcal{L}_{q}^{\mathrm{eff}}=\hat{\mathcal{L}}_{q}+\mathcal{L}_{q}^{\mathrm{osc}},
$$

where $\hat{\mathcal{L}}_{q}$ is the Hermitian Liouvillian in the rotating wave approximation shown in Eq. (33), where $\mathcal{O}\left(\hat{\mathcal{L}}_{q}\right)=\mathcal{O}\left(\sigma^{-1}\right)$, while $\mathcal{L}_{q}^{\text {osc }}$, of order $\mathcal{O}\left(\sigma^{0}\right)$, is composed by the oscillating terms that are neglected in the long-time limit. In particular, from Eq. (C4) one finds that the RWA holds for $t \gg \mathcal{O}\left(L^{2}\right)$. This approximation is therefore consistent with the results of Sec. IV, where one finds a Liouvillian gap $\mathcal{O}\left(\mathcal{L}^{-3}\right)$ that provides a lower bound to the convergence time $t>\mathcal{O}\left(L^{3}\right)$. However, while the eigenvalues depend only on the Hermitian operator $\hat{\mathcal{L}}_{q}$, the eigenvectors depend on the oscillating terms via Eq. (C6). By mixing Eq. (C5) with Eq. (D3), we find then that the eigenvalues with small $\mathcal{O}\left(\sigma^{-1}\right)$ real part have right eigenvectors given by

$$
e^{\mathcal{S}}\left|\Phi_{i}\right\rangle
$$

where $\left|\Phi_{i}\right\rangle$ form an orthonormal basis (dependent on both $H$ and $V), \mathcal{S} \approx \mathcal{S}_{\mathrm{RWA}}+\mathcal{S}_{D}+\left[\mathcal{S}_{\mathrm{RWA}}, \mathcal{S}_{D}\right] / 2=\mathcal{O}\left(\sigma^{-1}\right)$, but $e^{\mathcal{S} \dagger} \neq e^{-\mathcal{S}}$. The corresponding left eigenvectors are then $\left\langle\Phi_{i}\right| e^{-\mathcal{S}}$.

\section{APPENDIX E: DIAGONALIZATION OF THE RICHARDSON-GAUDIN MODEL}

We perform explicitly the diagonalization of the Richardson-Gaudin model Eq. (36) in the bosonic representation discussed in Sec. IVA, where $K_{i}^{-}=\tilde{a}_{i \uparrow} \tilde{a}_{i \downarrow}$, $K_{i}^{+}=\left(K_{i}^{-}\right)^{\dagger}$, and $K_{i}^{z}=\left(\tilde{n}_{i \uparrow}+\tilde{n}_{i \downarrow}+1\right) / 2$. We start by 
defining a trial eigenstate $\left|\Omega_{\nu}\right\rangle$ with no pairing, namely such that

$$
K_{i}^{-}\left|\Omega_{\nu}\right\rangle=0, \quad K_{i}^{z}\left|\Omega_{\nu}\right\rangle=\nu_{i}\left|\Omega_{\nu}\right\rangle .
$$

These equations force the constraints

$$
\nu_{i}=\left(n_{i \uparrow}+n_{i \downarrow}+1\right) / 2, \quad n_{i \uparrow} n_{i \downarrow}=0 ;
$$

namely, there cannot be in the same site both up particles and down particles. Moreover, $\nu_{0} \equiv 1 / 2$, because the model has been obtained by projecting the Liouvillian into the states where $n_{0 \uparrow}=n_{0 \downarrow}$. The eigenvalue of state $\left|\Omega_{\nu}\right\rangle$ is thus

$$
\begin{gathered}
\hat{\mathcal{L}_{q}}\left|\Omega_{\nu}\right\rangle=E_{0}\left|\Omega_{\nu}\right\rangle, \\
E_{0}=\frac{2}{\sigma}-\frac{8}{\sigma} \sum_{k} g_{k} \nu_{0} \nu_{k}=\frac{2}{\sigma}-\frac{4}{\sigma} \sum_{k} g_{k} \nu_{k} .
\end{gathered}
$$

Since there are extra constraints, $q=\sum_{i=0}^{L-1} n_{i \uparrow}=$ $\sum_{i=0}^{L-1} n_{i \downarrow}$, for a given set of allowed "quantum numbers" $v_{k}$, the number $N$ of paired particles satisfies $\sum_{i}\left(2 v_{i}-1\right)+2 N=2 q$; namely,

$$
N=q-\sum_{i}\left(v_{i}-\frac{1}{2}\right) .
$$

By defining the ansatz,

$$
|\psi\rangle=\prod_{\alpha=1}^{N} C_{\alpha}^{+}\left|\Omega_{\nu}\right\rangle, \quad C_{\alpha}^{+}=\sum_{j=0}^{L} u_{j \alpha} K_{j}^{+},
$$

one sees that

$$
\begin{aligned}
\hat{\mathcal{L}_{q}}|\psi\rangle= & E_{0}|\psi\rangle+\left[\hat{\mathcal{L}_{q}}, \prod_{\alpha} C_{\alpha}^{+}\right]\left|\Omega_{\nu}\right\rangle \\
= & E_{0}|\psi\rangle+\sum_{\alpha}\left(\prod_{\gamma \neq \alpha} C_{\gamma}^{+}\right)\left[\hat{\mathcal{L}}_{q}, C_{\alpha}^{+}\right]\left|\Omega_{\nu}\right\rangle \\
& +\frac{1}{2} \sum_{\alpha \neq \beta}\left(\prod_{\gamma \neq \alpha, \beta} C_{\gamma}^{+}\right)\left[\left[\hat{\mathcal{L}}_{q}, C_{\alpha}^{+}\right], C_{\beta}^{+}\right]\left|\Omega_{\nu}\right\rangle .
\end{aligned}
$$

Moreover,

$$
\begin{aligned}
{\left[\hat{\mathcal{L}}_{q}, C_{\alpha}^{+}\right] } & =-\frac{8}{\sigma} \sum_{k} g_{k}\left(u_{0 \alpha}-u_{k \alpha}\right)\left(K_{0}^{+} K_{k}^{z}-K_{0}^{z} K_{k}^{+}\right), \\
{\left[\left[\hat{\mathcal{L}}_{q}, C_{\alpha}^{+}\right], C_{\beta}^{+}\right] } & =\frac{8}{\sigma} \sum_{k} g_{k}\left(u_{0 \alpha}-u_{k \alpha}\right)\left(u_{0 \beta}-u_{k \beta}\right) K_{0}^{+} K_{k}^{+} .
\end{aligned}
$$

We now first consider the $N=1$ case and impose the eigenvalue equation $\hat{\mathcal{L}}_{q}|\psi\rangle=\lambda|\psi\rangle$, where we define
$\lambda=E_{0}-(8 / \sigma) \sum_{\alpha} E_{\alpha}$. The eigenvalue equation becomes then

$$
\begin{gathered}
\sum_{k} g_{k}\left(u_{0 \alpha}-u_{k \alpha}\right)\left(K_{0}^{+} \nu_{k}-\nu_{0} K_{k}^{+}\right) \\
=E_{\alpha}\left(u_{0 \alpha} K_{0}^{+}+\sum_{k} u_{k \alpha} K_{k}^{+}\right) .
\end{gathered}
$$

From that equation we get the relationship

$$
-\nu_{0} g_{k}\left(u_{0 \alpha}-u_{k \alpha}\right)=u_{k \alpha} E_{\alpha}
$$

namely,

$$
\begin{gathered}
u_{k \alpha}=\frac{\nu_{0} g_{k} u_{0 \alpha}}{\nu_{0} g_{k}-E_{\alpha}}, \\
u_{0 \alpha}-u_{k \alpha}=-\frac{E_{\alpha} u_{0 \alpha}}{\nu_{0} g_{k}-E_{\alpha}}=-\frac{E_{\alpha}}{\nu_{0} g_{k}} u_{k \alpha} .
\end{gathered}
$$

By using the last equation, we find

$$
\begin{aligned}
{\left[\left[\hat{\mathcal{L}}_{q}, C_{\alpha}^{+}\right], C_{\beta}^{+}\right] } & =\frac{8}{\sigma} \sum_{k} g_{k} \frac{E_{\alpha} u_{0 \alpha}}{\nu_{0} g_{k}-E_{\alpha}} \frac{E_{\beta} u_{0 \beta}}{\nu_{0} g_{k}-E_{\beta}} K_{0}^{+} K_{k}^{+} \\
& =\frac{8}{\sigma \nu_{0}} \frac{E_{\alpha} E_{\beta}}{E_{\alpha}-E_{\beta}} \sum_{k}\left(u_{0 \beta} u_{k \alpha}-u_{k \beta} u_{0 \alpha}\right) K_{0}^{+} K_{k}^{+} \\
& =\frac{8}{\sigma \nu_{0}} \frac{E_{\alpha} E_{\beta}}{E_{\alpha}-E_{\beta}}\left(u_{0 \beta} C_{\alpha}^{+}-C_{\beta}^{+} u_{0 \alpha}\right) K_{0}^{+} \\
& =\frac{8}{\sigma}\left(M_{\alpha \beta} K_{0}^{+} C_{\alpha}^{+}+M_{\beta \alpha} K_{0}^{+} C_{\beta}^{+}\right),
\end{aligned}
$$

where $M_{\alpha \beta}=\left(E_{\alpha} E_{\beta} / E_{\alpha}-E_{\beta}\right)\left(u_{0 \beta} / \nu_{0}\right)$. Using all the above results, the eigenvalue equation becomes

$$
\begin{array}{r}
\left(\hat{\mathcal{L}_{q}}-\lambda\right)|\psi\rangle=-\frac{8}{\sigma} \sum_{\alpha}\left(\prod_{\gamma \neq \alpha} C_{\gamma}^{+}\right) Z_{\alpha}\left|\Omega_{\nu}\right\rangle, \\
Z_{\alpha}=\sum_{k} \frac{g_{k} E_{\alpha} u_{0 \alpha}}{\nu_{0} g_{k}-E_{\alpha}}\left(\nu_{0} K_{k}^{+}-K_{0}^{+} \nu_{k}\right) \\
-\sum_{\beta \neq \alpha} M_{\beta \alpha} K_{0}^{+}-E_{\alpha}\left(u_{0 \alpha} K_{0}^{+}+\sum_{k} u_{k \alpha} K_{k}^{+}\right) .
\end{array}
$$

By evaluating $Z_{\alpha}=0$, one gets the equations

$$
\sum_{k} \frac{g_{k} \nu_{k} E_{\alpha}}{E_{\alpha}-\nu_{0} g_{k}}+\frac{1}{\nu_{0}} \sum_{\beta \neq \alpha} \frac{E_{\alpha} E_{\beta}}{E_{\alpha}-E_{\beta}}=E_{\alpha},
$$

for $\alpha=1, \ldots, N$, where $N$ is given by Eq. (E5). Clearly, $E_{\alpha}=0$ is a solution, while the solutions different from zero are found by solving the equation 


$$
\sum_{k} \frac{2 g_{k} \nu_{k}}{2 E_{\alpha}-g_{k}}+2 \sum_{\beta \neq \alpha} \frac{E_{\beta}}{E_{\alpha}-E_{\beta}}=1
$$

where we use the fact that $\nu_{0}=1 / 2$. In conclusion, the eigenvalues of the Liouvillian $\hat{\mathcal{L}_{q}}$ are

$$
\lambda=E_{0}-\frac{8}{\sigma} \sum_{\alpha} E_{\alpha}=-\frac{2}{\sigma}\left(\sum_{k} g_{k} n_{k}+4 \sum_{\alpha=1}^{q-\sum_{k} n_{k} / 2} E_{\alpha}\right),
$$

where $n_{k}=2 \nu_{k}-1$ and the $E_{\alpha}$ are either zero or the solution of Eq. (E15). From that expression it is clear that the steady state corresponds to $E_{\alpha}=0$ and $n_{k}=0$. The eigenvalues for larger values of $q$ are given by all the previous solutions with smaller $q$ (this can be seen by adding some $E_{\beta}=0$ for larger values of $N$ ) together with new solutions due to the larger values of $N$ and the larger set of allowed configurations for $n_{k}$.

\section{APPENDIX F: SOLUTION OF THE SU(2q)-INVARIANT GAUDIN MODEL}

We describe here the algebraic approach to general Gaudin models and then apply it to our general fermionic representation introduced in Sec. IV C. We fix a basis $h_{\alpha}^{(j)}$ of the Cartan subalgebra acting on the $j$ th copy formed by the diagonal operators $X_{\alpha \alpha}^{(j)}$. A state $\left|\Omega_{\nu}\right\rangle$ which is a simultaneous eigenvector of all the operators $h_{\alpha}^{(j)}$ is called a weight vector. We write $h_{\alpha}^{(j)}\left|\Omega_{\nu}\right\rangle=\nu_{j \alpha}\left|\Omega_{\nu}\right\rangle$, where $\nu_{\alpha}^{j}$ is called weight. On the other hand, in the Cartan-Weyl basis the eigenvalue $\chi$ of the adjoint transformation, namely, $\left[h_{\alpha}^{(j)}, e_{\chi}\right]=\chi_{j \alpha} e_{\chi}$, for a given $e_{\chi}$ in the representation, is called a root. Because of Eq. (44) a root can only have eigenvalue $-1,0,1$. If one fixes an ordering $c_{\alpha}>c_{\alpha+1}$ and writes $h^{(j)}=\sum_{\alpha} c_{\alpha} h_{\alpha}^{(j)}$, then the eigenoperators of $h^{(j)}$ with positive eigenvalue are called the "raising operators." They correspond to $X_{\alpha \beta}^{(j)}$ for any $\alpha<\beta$. A highest weight vector is a weight vector $\left|\Omega_{\nu}\right\rangle$ such that all the other vectors in an irreducible representation can be obtained from $\left|\Omega_{\nu}\right\rangle$ via some lowering operators. As such, a highest weight vector is annihilated by all the raising operators. We call $\chi^{j}$ the simple roots of the algebra, and we fix an inner product between roots $\left(\chi^{j}, \chi^{k}\right)=\sum_{\alpha} \chi_{\alpha}^{j} \chi_{\alpha}^{k}$, and write $\left|\chi^{j}\right|^{2}=\left(\chi^{j}, \chi^{j}\right)$. The matrix $C_{j k}=\left[2\left(\chi^{j}, \chi^{k}\right) /\left|\chi^{j}\right|^{2}\right]$ is called the Cartan matrix. We also call $F_{j k}=\left(C^{-1}\right)_{j k}\left(2 /\left|\chi^{k}\right|^{2}\right)$. Moreover, we call $z_{0}=0$ and $z_{k}=2 g_{k}^{-1}$ for $k>0$.

Thanks to the above definitions, and owing to the results of Refs. [63,64], we can write the eigenvalues of the Gaudin model Eq. (43) as

$$
\begin{aligned}
\lambda_{\{\mu\}}= & -\frac{2 q}{\sigma}+\frac{8}{\sigma}\left[\sum_{k=1}^{L-1} \frac{\sum_{i j} \mu_{i}^{0} F_{i j} \mu_{j}^{k}}{z_{k}-z_{0}}+\right. \\
& \left.+\sum_{j=1}^{2 q-1} \sum_{\alpha} \frac{\left|\chi^{j}\right|^{2}}{2} \frac{\mu_{j}^{0}}{z_{0}-\omega_{j \alpha}}\right],
\end{aligned}
$$

where $\mu_{k}^{j}$ are the eigenvalues of the Chevalley operators $H_{k}^{(j)}=\left(2 /\left|\chi^{k}\right|^{2}\right) \sum_{\alpha} \chi_{\alpha}^{k} h_{\alpha}^{(j)}$, namely, $H_{k}^{(j)}\left|\Omega_{\nu}\right\rangle=\mu_{k}^{j}\left|\Omega_{\nu}\right\rangle$ and so $\mu_{k}^{j}=\left(2 /\left|\chi^{k}\right|^{2}\right) \sum_{\alpha} \chi_{\alpha}^{k} \nu_{k}^{j}$, and where the Bethe roots satisfy the equations

$$
\sum_{i \beta} \frac{C_{i j}}{\omega_{i \beta}-\omega_{j \alpha}}=\sum_{k=0}^{L-1} \frac{\mu_{j}^{k}}{z_{k}-\omega_{j \alpha}} .
$$

The above expressions for the eigenvalues hold whenever the operators $X_{\alpha \beta}^{(j)}$ define any semisimple Lie algebra. In the particular case discussed in Sec. IV C, those operators define a $\mathrm{SU}(2 q)$-invariant Gaudin model, in a specific multifermion representation. For $\mathrm{SU}(2 q)$, the simple roots are $\chi_{\alpha}^{j}=\delta_{\alpha j}-\delta_{\alpha+1, j}$, so $\left|\chi^{j}\right|^{2}=2$, and $C_{i j}=2 \delta_{i j}-\left(\delta_{i, j-1}+\delta_{i, j+1}\right)$, where $i, j=1, \ldots, 2 q-1$. Therefore, $\quad F_{i j}=\sum_{\ell}(2 / 2 q)[\sin (\pi i \ell / 2 q) \sin (\pi j \ell / 2 q)] /$ $[2-2 \cos (\ell \pi / 2 q)]$, and the Chevalley operators are given by $H_{\alpha}^{(j)}=X_{\alpha, \alpha}^{(j)}-X_{\alpha+1, \alpha+1}^{(j)}$. We fix the ordering $\{(\downarrow, 1)$, $(\downarrow, 2), \ldots,(\uparrow, 1),(\uparrow, 2), \ldots\}$ so that

$H_{\alpha}^{j}= \begin{cases}-\tilde{a}_{j, \alpha, \downarrow}^{\dagger} \tilde{a}_{j, \alpha, \downarrow}+\tilde{a}_{j, \alpha+1, \downarrow}^{\dagger} \tilde{a}_{j, \alpha+1, \downarrow} & \text { for } 1 \leq \alpha \leq q-1 \\ \tilde{a}_{j, \alpha, \uparrow}^{\dagger} \tilde{a}_{j, \alpha, \uparrow}-\tilde{a}_{j, \alpha+1, \uparrow}^{\dagger} \tilde{a}_{j, \alpha+1, \uparrow} & \text { for } 1 \leq \alpha-q \leq q-1 \\ 1-\tilde{a}_{j, q, \downarrow}^{\dagger} \tilde{a}_{j, q, \downarrow}-\tilde{a}_{j, 1, \uparrow}^{\dagger} \tilde{a}_{j, 1, \uparrow} & \text { for } \alpha=q .\end{cases}$

Because of the above equations, the raising operators are given by $a_{j, i, \uparrow}^{\dagger} a_{j, k, \uparrow}$, with $i>k$, by $a_{j, i, \downarrow}^{\dagger} a_{j, k, \downarrow}$ with $i<k$, and by $a_{j, i, \sigma} a_{j, k, \sigma}$. Therefore, the highest weight vectors may contain in the same mode $j$ either spin- $\uparrow$ particles or spin- $\downarrow$ particles, but not both. The only possible highest weight states are then either $\prod_{i}^{n_{j \uparrow}} a_{j, i, \uparrow}^{\dagger}|0\rangle$ or $\prod_{i}^{n_{j \downarrow}} a_{j, q-i+1, \downarrow}^{\dagger}|0\rangle$. These states are parametrized by the numbers $n_{j \uparrow}$ and $n_{j \downarrow}$ that satisfy $n_{j \uparrow} n_{j \downarrow}=0$. Therefore,

$\mu_{j}^{k}=\delta_{j, q}\left(1-\delta_{n_{\downarrow k}>0}-\delta_{n_{\uparrow k}>0}\right)+\delta_{j, q+n_{\uparrow k}}+\delta_{j, q-n_{\downarrow k}}$.

Moreover, $n_{0 \uparrow}=n_{0 \downarrow}$, so $\mu_{j}^{0}=\delta_{j, q}$. By explicit calculation for $j \leq q$, one finds $F_{q j}=F_{q, 2 q-j}=j / 2$. Therefore, Eq. (F1) becomes 


$$
\begin{aligned}
\lambda_{\{n\}}= & -\frac{2 q}{\sigma}+\frac{8}{\sigma}\left[\sum _ { k = 1 } ^ { L - 1 } \frac { g _ { k } } { 2 } \left(\frac{q}{2}\left(1-\delta_{n_{\downarrow k}>0}-\delta_{n_{\uparrow k}>0}\right)\right.\right. \\
& \left.+\frac{\left(q-n_{\downarrow k}\right) \delta_{n_{\downarrow k}>0}+\left(q-n_{\uparrow k}\right) \delta_{n_{\uparrow k}>0}}{2}\right) \\
& \left.-\sum_{\alpha} \frac{1}{\omega_{q, \alpha}}\right] \\
= & -\frac{2}{\sigma}\left[\sum_{k=1}^{L-1} g_{k}\left(n_{\downarrow k}+n_{\uparrow k}\right)+4 \sum_{\alpha} \frac{1}{\omega_{q, \alpha}}\right],
\end{aligned}
$$

where $\omega_{j, \alpha}$ are the solutions of Eq. (F2), namely, of Eq. (47).

\section{APPENDIX G: EXPLICIT MEAN-FIELD ANALYSIS}

In this section, we perform explicitly the mean-field calculations discussed in Sec. III B, and we closely follow the notation of that section. We remind the reader that Eq. (28) can be written as

$$
\mathcal{L}_{q}=\sum_{i} \lambda_{i} \tilde{a}_{i}^{\prime} \tilde{a}_{i}-\frac{\sigma}{2} \sum_{i, j, k, l} \tilde{V}_{i j} \tilde{V}_{k l} \tilde{a}_{i}^{\prime} \tilde{a}_{k}^{\prime} \tilde{a}_{j} \tilde{a}_{l},
$$

where the $\lambda$ 's are ordered with decreasing (negative) real part, $\lambda_{0}=0, \stackrel{\circ}{V}=Z \tilde{V} Z^{-1}$, and we remind the reader that the new bosonic creation operators are obtained via the nonunitary Bogoliubov transformation $\tilde{a}_{i}^{\prime}=\sum_{\alpha} Z_{\alpha i} a_{\alpha}^{\dagger}$, $\tilde{a}_{i}=\sum_{\alpha}\left(Z^{-1}\right)_{i \alpha} a_{\alpha}$. The steady state is therefore the boson condensate $|\Omega\rangle=\left[\left(\tilde{a}_{0}^{\prime}\right)^{q} / \sqrt{q} !\right]|0\rangle$, where $|0\rangle$ is the bosonic vacuum. Indeed, clearly this state is annihilated by the quadratic term. To see that even the second one annihilates, it is important to remember that $S_{0 \alpha}^{-1}$ is the right eigenvector of the steady state (corresponding to the steady state) and the corresponding left eigenvalue $S_{\alpha 0}$ is the identity operator. Therefore, $\quad \tilde{V}_{i 0}=\sum_{\alpha \beta} S_{j \alpha}^{-1} \stackrel{\circ}{V}_{\alpha \beta} S_{\beta 0}=0$, since $\sum_{\beta} \stackrel{\circ}{V}_{\alpha \beta} S_{\beta 0}$ is a vectorization of the expression $[V, \mathbb{1}]$. Similarly, $\tilde{V}_{0 i}=0$. To study the elementary excitations with respect to this state, one can use the Bogoliubov (mean-field) approach starting from the variational states $|\psi\rangle=\sum_{j} \psi_{j}\left[\left(\tilde{a}_{0}^{\prime}\right)^{q-1} / \sqrt{(q-1) !}\right] a_{j}^{\prime}|0\rangle$, for $j \neq 0$, and the corresponding $\left\langle\psi^{\prime}\right|=\sum_{j} \psi_{j}^{\prime}\langle 0|\left[\left(\tilde{a}_{0}\right)^{q-1} / \sqrt{(q-1) !}\right] a_{j}$, where $\sum_{j} \psi_{j}^{\prime} \psi_{j}=1$. The variational Liouvillian then becomes

$$
\begin{aligned}
\mathcal{L}^{V} & =\left\langle\psi^{\prime}\left|\mathcal{L}_{q}\right| \psi\right\rangle \\
& =\sum_{j} \lambda_{j} \psi_{j}^{\prime} \psi_{j}-\frac{\sigma}{2} \sum_{i, j, k, l} \tilde{V}_{i j} \tilde{V}_{k l}\left\langle\psi^{\prime}\left|\tilde{a}_{i}^{\prime} \tilde{a}_{k}^{\prime} \tilde{a}_{j} \tilde{a}_{l}\right| \psi\right\rangle,
\end{aligned}
$$

which, similarly to the Rayleigh-Ritz method, has to satisfy $\partial_{\psi} \mathcal{L}^{V}=\partial_{\psi^{\prime}} \mathcal{L}^{V}=0$ with the constraint $\sum_{j} \psi_{j}^{\prime} \psi_{j}=1$ (see, e.g., Ref. [50]). However, because $\tilde{V}_{i 0}=\tilde{V}_{0 i}=0$ for all $i$, one can restrict the sum in the above equation to the values $i, j, k, l>0$, but because there is only one particle in $|\psi\rangle$ in the states $i>0$, one finds that

$$
\mathcal{L}^{V}=\sum_{j} \lambda_{j} \psi_{j}^{\prime} \psi_{j}
$$

namely, that in the single-excitation subspace the variational Liouvillian is already diagonal. This shows that the eigenvalues, at least in the low-energy subspace, are not "renormalized" for larger values of $q$.

[1] T. Guhr, A. Müller-Groeling, and H. A. Weidenmüller, Random-Matrix Theories in Quantum Physics: Common Concepts, Phys. Rep. 299, 189 (1998).

[2] P. Hayden, D. Leung, P. W. Shor, and A. Winter, Randomizing Quantum States: Constructions and Applications, Commun. Math. Phys. 250, 371 (2004).

[3] A. Bendersky, F. Pastawski, and J. P. Paz, Selective and Efficient Estimation of Parameters for Quantum Process Tomography, Phys. Rev. Lett. 100, 190403 (2008).

[4] C. Dankert, R. Cleve, J. Emerson, and E. Livine, Exact and Approximate Unitary 2-Designs and Their Application to Fidelity Estimation, Phys. Rev. A 80, 012304 (2009).

[5] A. Harrow, P. Hayden, and D. Leung, Superdense Coding of Quantum States, Phys. Rev. Lett. 92, 187901 (2004).

[6] A. Abeyesinghe, I. Devetak, P. Hayden, and A. Winter, in Proceedings of the Royal Society of London A: Mathematical, Physical and Engineering Sciences (The Royal Society, London, 2009), p. rspa20090202.

[7] M. B. Hastings, Superadditivity of Communication Capacity Using Entangled Inputs, Nat. Phys. 5, 255 (2009).

[8] D. P. DiVincenzo, D. W. Leung, and B. M. Terhal, Quantum Data Hiding, IEEE Trans. Inf. Theory 48, 580 (2002).

[9] M. Piani, V. Narasimhachar, and J. Calsamiglia, Quantumness of Correlations, Quantumness of Ensembles and Quantum Data Hiding, New J. Phys. 16, 113001 (2014).

[10] R. Oliveira, O. C. O. Dahlsten, and M. B. Plenio, Generic Entanglement Can Be Generated Efficiently, Phys. Rev. Lett. 98, 130502 (2007).

[11] M. Žnidarič, Exact Convergence Times for Generation of Random Bipartite Entanglement, Phys. Rev. A 78, 032324 (2008).

[12] A. Hamma, S. Santra, and P. Zanardi, Quantum Entanglement in Random Physical States, Phys. Rev. Lett. 109, 040502 (2012).

[13] P. Zanardi, Local Random Quantum Circuits: Ensemble Completely Positive Maps and Swap Algebras, J. Math. Phys. (N.Y.) 55, 082204 (2014).

[14] F. G. S. L. Brandao, A. W. Harrow, and M. Horodecki, Local Random Quantum Circuits Are Approximate Polynomial-Designs, Commun. Math. Phys. 346, 397 (2016). 
[15] W. G. Brown and L. Viola, Convergence Rates for Arbitrary Statistical Moments of Random Quantum Circuits, Phys. Rev. Lett. 104, 250501 (2010).

[16] J. Emerson, Y. S. Weinstein, M. Saraceno, S. Lloyd, and D. G. Cory, Pseudo-Random Unitary Operators for Quantum Information Processing, Science 302, 2098 (2003).

[17] D. Gross, K. Audenaert, and J. Eisert, Evenly Distributed Unitaries: On the Structure of Unitary Designs, J. Math. Phys. (N.Y.) 48, 052104 (2007).

[18] A. W. Harrow and R. A. Low, Approximation, Randomization, and Combinatorial Optimization. Algorithms and Techniques (Springer, New York, 2009), pp. 548-561.

[19] P. S. Turner and D. Markham, Derandomizing Quantum Circuits with Measurement-Based Unitary Designs, Phys. Rev. Lett. 116, 200501 (2016).

[20] R. N. Alexander, P. S. Turner, and S. D. Bartlett, Randomized Benchmarking in Measurement-Based Quantum Computing, Phys. Rev. A 94, 032303 (2016).

[21] A. Perez-Leija, R. Keil, A. Kay, H. Moya-Cessa, S. Nolte, L.-C. Kwek, B. M. Rodríguez-Lara, A. Szameit, and D. N. Christodoulides, Coherent Quantum Transport in Photonic Lattices, Phys. Rev. A 87, 012309 (2013).

[22] I. Pitsios, L. Banchi, A. S. Rab, M. Bentivegna, D. Caprara, A. Crespi, N. Spagnolo, S. Bose, P. Mataloni, R. Osellame et al., Photonic Simulation of Entanglement Growth after a Spin Chain Quench, arXiv:1603.02669.

[23] J. Carolan, C. Harrold, C. Sparrow, E. Martín-López, N. J. Russell, J. W. Silverstone, P. J. Shadbolt, N. Matsuda, M. Oguma, M. Itoh et al., Universal Linear Optics, Science 349, 711 (2015).

[24] M. A. Broome, A. Fedrizzi, S. Rahimi-Keshari, J. Dove, S. Aaronson, T. C. Ralph, and A. G. White, Photonic Boson Sampling in a Tunable Circuit, Science 339, 794 (2013).

[25] J. B. Spring, B. J. Metcalf, P. C. Humphreys, W. Steven Kolthammer, X.-M. Jin, M. Barbieri, A. Datta, N. Thomas-Peter, N. K. Langford, D. Kundys et al., Boson Sampling on a Photonic Chip, Science 339, 798 (2013).

[26] A. Crespi, R. Osellame, R. Ramponi, D. J. Brod, E. F. Galvão, N. Spagnolo, C. Vitelli, E. Maiorino, P. Mataloni, and F. Sciarrino, Integrated Multimode Interferometers with Arbitrary Designs for Photonic Boson Sampling, Nat. Photonics 7, 545 (2013).

[27] S. Aaronson and A. Arkhipov, in Proceedings of the FortyThird Annual ACM Symposium on Theory of Computing (Association for Computing Machinery, New York, 2011), pp. 333-342.

[28] P. Hayden and J. Preskill, Black Holes as Mirrors: Quantum Information in Random Subsystems, J. High Energy Phys. 09 (2007) 120.

[29] Y. Sekino and L. Susskind, Fast Scramblers, J. High Energy Phys. 10 (2008) 065.

[30] N. Lashkari, D. Stanford, M. Hastings, T. Osborne, and P. Hayden, Towards the Fast Scrambling Conjecture, J. High Energy Phys. 04 (2013) 022.

[31] D. D'Alessandro, Introduction to Quantum Control and Dynamics (CRC Press, Boca Raton, FL, 2007).
[32] V. Jurdjevic and H. J. Sussmann, Control Systems on Lie Groups, J. Differ. Equations 12, 313 (1972).

[33] Y. Nakata, C. Hirche, M. Koashi, and A. Winter, Efficient Quantum Pseudorandomness with Nearly TimeIndependent Hamiltonian Dynamics, Phys. Rev. X 7, 021006 (2017).

[34] E. Onorati, O. Buerschaper, M. Kliesch, W. Brown, A. H. Werner, and J. Eisert, Mixing Properties of Stochastic Quantum Hamiltonians, Commun. Math. Phys. 355, 905 (2017).

[35] F. G. S. L. Brandao, A. W. Harrow, and M. Horodecki, Local Random Quantum Circuits Are Approximate Polynomial-Designs, Commun. Math. Phys. 346, 397 (2016).

[36] R. A. Low, Pseudo-Randomness and Learning in Quantum Computation, Ph.D. thesis, University of Bristol, 2010.

[37] R. I. Oliveira, On the Convergence to Equilibrium of Kac's Random Walk on Matrices, Ann. Appl. Probab. 19, 1200 (2009).

[38] A. Ishizaki and G. R. Fleming, Unified Treatment of Quantum Coherent and Incoherent Hopping Dynamics in Electronic Energy Transfer: Reduced Hierarchy Equation Approach, J. Chem. Phys. 130, 234111 (2009).

[39] L. Banchi, G. Costagliola, A. Ishizaki, and P. Giorda, An Analytical Continuation Approach for Evaluating Emission Lineshapes of Molecular Aggregates and the Adequacy of Multichromophoric Förster Theory, J. Chem. Phys. 138, 184107 (2013).

[40] G. Lindblad, On the Generators of Quantum Dynamical Semigroups, Commun. Math. Phys. 48, 119 (1976).

[41] V. Gorini and A. Kossakowski, N-Level System in Contact with a Singular Reservoir, J. Math. Phys. (N.Y.) 17, 1298 (1976).

[42] M. Mézard, G. Parisi, and M. Virasoro, Spin Glass Theory and Beyond: An Introduction to the Replica Method and Its Applications (World Scientific Publishing Co, Inc, Singapore, 1987), Vol. 9.

[43] V. V. Albert and L. Jiang, Symmetries and Conserved Quantities in Lindblad Master Equations, Phys. Rev. A 89, 022118 (2014).

[44] R. Goodman and N. R. Wallach, Representations and Invariants of the Classical Groups, Vol. 68 (Cambridge University Press, Cambridge, England, 2000).

[45] F. G. S. L. Brandão, P. Ćwikliński, M. Horodecki, P. Horodecki, J. K. Korbicz, and M. Mozrzymas, Convergence to Equilibrium under a Random Hamiltonian, Phys. Rev. E 86, 031101 (2012).

[46] P. Zanardi, J. Marshall, and L. C. Venuti, Dissipative Universal Lindbladian Simulation, Phys. Rev. A 93, 022312 (2016).

[47] M. J. Kastoryano, D. Reeb, and M. M. Wolf, A Cutoff Phenomenon for Quantum Markov Chains, J. Phys. A 45, 075307 (2012).

[48] J.-P. Blaizot and G. Ripka, Quantum Theory of Finite Systems, Vol. 3 (MIT Press, Cambridge, MA, 1986).

[49] D. J. Rowe, M. J. Carvalho, and J. Repka, Dual Pairing of Symmetry and Dynamical Groups in Physics, Rev. Mod. Phys. 84, 711 (2012). 
[50] A. Laestadius and S. Kvaal, Analysis of the Extended Coupled-Cluster Method in Quantum Chemistry, arXiv: 1702.04317.

[51] R. Zeier and T. Schulte-Herbrüggen, Symmetry Principles in Quantum Systems Theory, J. Math. Phys. (N.Y.) 52, 113510 (2011).

[52] Z. Zimborás, R. Zeier, T. Schulte-Herbrüggen, and D. Burgarth, Symmetry Criteria for Quantum Simulability of Effective Interactions, Phys. Rev. A 92, 042309 (2015).

[53] M. Obata, On Subgroups of the Orthogonal Group, Trans. Am. Math. Soc. 87, 347 (1958).

[54] G. M. Nikolopoulos, I. Jex et al., Quantum State Transfer and Network Engineering (2014), http://www.springer.com/ us/book/9783642399367.

[55] L. Banchi, T. J. G. Apollaro, A. Cuccoli, R. Vaia, and P. Verrucchi, Long Quantum Channels for High-Quality Entanglement Transfer, New J. Phys. 13, 123006 (2011).

[56] D. Burgarth and S. Bose, Conclusive and Arbitrarily Perfect Quantum-State Transfer Using Parallel Spin-Chain Channels, Phys. Rev. A 71, 052315 (2005).

[57] L. Banchi, A. Bayat, P. Verrucchi, and S. Bose, Nonperturbative Entangling Gates between Distant Qubits Using Uniform Cold Atom Chains, Phys. Rev. Lett. 106, 140501 (2011).

[58] X. Wang, P. Pemberton-Ross, and S. G. Schirmer, Symmetry and Subspace Controllability for Spin Networks with a Single-Node Control, IEEE Trans. Autom. Control 57, 1945 (2012).

[59] D. Burgarth, D. D’Alessandro, L. Hogben, S. Severini, and M. Young, Zero Forcing, Linear and Quantum Controllability for Systems Evolving on Networks, IEEE Trans. Autom. Control 58, 2349 (2013).

[60] M. Gaudin, Diagonalisation d'une Classe d'Hamiltoniens de Spin, J. Phys. (Paris) 37, 1087 (1976).

[61] R. W. Richardson, Exactly Solvable Many-Boson Model, J. Math. Phys. (N.Y.) 9, 1327 (1968).

[62] G. Szegö, in Orthogonal Polynomials, American Mathematical Society Colloquium Vol. 23 (American Mathematical Society, Providence, 1959).

[63] A. G. Ushveridze, Quasi-Exactly Solvable Models in Quantum Mechanics (CRC Press, Boca Raton, FL, 1994).

[64] F. Falceto and K. Gawędzki, Unitarity of the KnizhnikZamolodchikov-Bernard Connection and the Bethe Ansatz for the Elliptic Hitchin Systems, Commun. Math. Phys. 183, 267 (1997).

[65] E. Mukhin, V. Tarasov, and A. Varchenko, Bispectral and $\left(\mathrm{gl}_{\mathrm{N}}, \mathrm{gl}_{\mathrm{M}}\right)$ Dualities, Discrete versus Differential, Adv. Math. 218, 216 (2008).

[66] B. Feigin, E. Frenkel, and N. Reshetikhin, Gaudin Model, Bethe Ansatz and Critical Level, Commun. Math. Phys. 166, 27 (1994).
[67] J. M. Roman, G. Sierra, and J. Dukelsky, Large-N Limit of the Exactly Solvable BCS Model: Analytics versus Numerics, Nucl. Phys. B634, 483 (2002).

[68] M. Žnidarič, Relaxation Times of Dissipative Many-Body Quantum Systems, Phys. Rev. E 92, 042143 (2015).

[69] C. Spengler, M. Huber, and B. C. Hiesmayr, Composite Parameterization and Haar Measure for All Unitary and Special Unitary Groups, J. Math. Phys. (N.Y.) 53, 013501 (2012).

[70] E. Lieb, T. Schultz, and D. Mattis, Two Soluble Models of an Antiferromagnetic Chain, Ann. Phys. (N.Y.) 16, 407 (1961).

[71] J. R. Johansson, P. D. Nation, and F. Nori, QuTip: An Open-Source PYTHON Framework for the Dynamics of Open Quantum Systems, Comput. Phys. Commun. 183, 1760 (2012).

[72] A. Pal and D. A. Huse, Many-Body Localization Phase Transition, Phys. Rev. B 82, 174411 (2010).

[73] P. Ponte, Z. Papić, F. Huveneers, and D. A. Abanin, Many-Body Localization in Periodically Driven Systems, Phys. Rev. Lett. 114, 140401 (2015).

[74] M. V. Medvedyeva, F. H. L. Essler, and T. Prosen, Exact Bethe Ansatz Spectrum of a Tight-Binding Chain with Dephasing Noise, Phys. Rev. Lett. 117, 137202 (2016).

[75] L. Banchi, P. Giorda, and P. Zanardi, Quantum InformationGeometry of Dissipative Quantum Phase Transitions, Phys. Rev. E 89, 022102 (2014).

[76] S. Diehl, E. Rico, M. A. Baranov, and P. Zoller, Topology by Dissipation in Atomic Quantum Wires, Nat. Phys. 7, 971 (2011).

[77] T. Prosen and I. Pižorn, Quantum Phase Transition in a Farfrom-Equilibrium Steady State of an XY Spin Chain, Phys. Rev. Lett. 101, 105701 (2008).

[78] A. Nahum, J. Ruhman, S. Vijay, and J. Haah, Quantum Entanglement Growth under Random Unitary Dynamics, Phys. Rev. X 7, 031016 (2017).

[79] J. Eisert, M. Friesdorf, and C. Gogolin, Quantum ManyBody Systems Out of Equilibrium, Nat. Phys. 11, 124 (2015).

[80] P. Hayden, D. W. Leung, and A. Winter, Aspects of Generic Entanglement, Commun. Math. Phys. 265, 95 (2006).

[81] M. M. Wolf, Quantum Channels and Operations Guided Tour, http://www-m5.ma.tum.de/foswiki/pub/M5/ Allgemeines/MichaelWolf/QChannelLecture.pdf.

[82] C. Fleming, N. I. Cummings, C. Anastopoulos, and B. L. $\mathrm{Hu}$, The Rotating-Wave Approximation: Consistency and Applicability from an Open Quantum System Analysis, J. Phys. A 43, 405304 (2010).

[83] P. Zanardi and L. C. Venuti, Coherent Quantum Dynamics in Steady-State Manifolds of Strongly Dissipative Systems, Phys. Rev. Lett. 113, 240406 (2014). 